\title{
Spectral analysis of the transition to turbulence from a dipole in stratified fluid
}

\author{
Pierre Augier $\dagger$, Jean-Marc Chomaz and Paul Billant \\ LadHyX, CNRS, Ecole Polytechnique, 91128 Palaiseau CEDEX, France
}

(Received 24 December 2011; revised 11 July 2012; accepted 31 August 2012; first published online 11 October 2012)

We investigate the spectral properties of the turbulence generated during the nonlinear evolution of a Lamb-Chaplygin dipole in a stratified fluid for a high Reynolds number $R e=28000$ and a wide range of horizontal Froude number $F_{h} \in\left[\begin{array}{ll}0.0225 & 0.135] \text { and }\end{array}\right.$ buoyancy Reynolds number $\mathscr{R}=\operatorname{ReF}_{h}{ }^{2} \in\left[\begin{array}{ll}14 & 510\end{array}\right]$. The numerical simulations use a weak hyperviscosity and are therefore almost direct numerical simulations (DNS). After the nonlinear development of the zigzag instability, both shear and gravitational instabilities develop and lead to a transition to small scales. A spectral analysis shows that this transition is dominated by two kinds of transfer: first, the shear instability induces a direct non-local transfer toward horizontal wavelengths of the order of the buoyancy scale $L_{b}=U / N$, where $U$ is the characteristic horizontal velocity of the dipole and $N$ the Brunt-Väisälä frequency; second, the destabilization of the Kelvin-Helmholtz billows and the gravitational instability lead to small-scale weakly stratified turbulence. The horizontal spectrum of kinetic energy exhibits a $\varepsilon_{K}^{2 / 3} k_{h}^{-5 / 3}$ power law (where $k_{h}$ is the horizontal wavenumber and $\varepsilon_{K}$ is the dissipation rate of kinetic energy) from $k_{b}=2 \pi / L_{b}$ to the dissipative scales, with an energy deficit between the integral scale and $k_{b}$ and an excess around $k_{b}$. The vertical spectrum of kinetic energy can be expressed as $E\left(k_{z}\right)=C_{N} N^{2} k_{z}^{-3}+C \varepsilon_{K}{ }^{2 / 3} k_{z}^{-5 / 3}$ where $C_{N}$ and $C$ are two constants of order unity and $k_{z}$ is the vertical wavenumber. It is therefore very steep near the buoyancy scale with an $N^{2} k_{z}^{-3}$ shape and approaches the $\varepsilon_{K}^{2 / 3} k_{z}^{-5 / 3}$ spectrum for $k_{z}>k_{o}, k_{o}$ being the Ozmidov wavenumber, which is the cross-over between the two scaling laws. A decomposition of the vertical spectra depending on the horizontal wavenumber value shows that the $N^{2} k_{z}^{-3}$ spectrum is associated with large horizontal scales $\left|\boldsymbol{k}_{h}\right|<k_{b}$ and the $\varepsilon_{K}^{2 / 3} k_{z}^{-5 / 3}$ spectrum with the scales $\left|\boldsymbol{k}_{h}\right|>k_{b}$.

Key words: instability, stratified flows, transition to turbulence

\section{Introduction}

Our understanding of the dynamics of strongly stratified flows has taken a major step forward with the realization of the importance of the anisotropy and of the 'buoyancy' scaling law which states that the vertical length scale of a structure should scale as the buoyancy length scale $L_{b}=U / N$, where $U$ is the typical velocity of

$\dagger$ Present adress: Linné Flow Centre, KTH Mechanics, KTH, SE-100 44 Stockholm, Sweden. Email address for correspondence: augier@ladhyx.polytechnique.fr 
the structure and $N$ is the Brunt-Väisälä frequency. This scaling law is valid in the inviscid limit when the horizontal Froude number $F_{h}=U /\left(N L_{h}\right)$ (where $L_{h}$ is the typical horizontal length scale) is small and implies that the potential energy is of the same order as the kinetic energy. Theoretically, it comes from the invariance of the Boussinesq-Euler equations under the hydrostatic approximation with respect to variation of the stratification (Billant \& Chomaz 2001).

From the turbulence point of view, this scaling law leads to the hypothesis of a direct energy cascade (Lindborg 2002, 2006). Such a cascade and the importance of the buoyancy length scale in strongly stratified turbulence has been observed in many numerical studies (Godeferd \& Staquet 2003; Laval, McWilliams \& Dubrulle 2003; Riley \& de Bruyn Kops 2003; Waite \& Bartello 2004; Hebert \& de Bruyn Kops 2006; Lindborg 2006; Brethouwer et al. 2007; Lindborg \& Brethouwer 2007).

When the horizontal Froude number is small and the buoyancy Reynolds number $\mathscr{R}=R e F_{h}^{2}$ is very large (where $R e$ is the usual Reynolds number, $R e=U L_{h} / v$, with $v$ the viscosity), there exists a universal regime of strongly stratified turbulence associated with a horizontal kinetic energy spectrum of the form $C_{1} \varepsilon_{K}{ }^{2 / 3} k_{h}^{-5 / 3}$, where $k_{h}$ is the horizontal wavenumber and $\varepsilon_{K}$ is the dissipation rate of kinetic energy, and with $C_{1}=0.5$ a universal constant (Lindborg 2006; Brethouwer et al. 2007).

This $k_{h}^{-5 / 3}$ horizontal energy spectrum for strongly stratified turbulence is followed by a weakly stratified cascade at small scales (Brethouwer et al. 2007). The strongly stratified inertial range has been predicted to exhibit vertical spectra of the form $N^{2} k_{z}^{-3}$, where $k_{z}$ is the vertical wavenumber. On the contrary, the weakly stratified cascade is nearly isotropic and thus associated with a $\varepsilon_{K}{ }^{2 / 3} k_{z}^{-5 / 3}$ vertical spectrum. The transition between the two regimes happens at the Ozmidov length scale $l_{o}=\sqrt{\varepsilon_{K} / N^{3}}$ (Lumley 1964; Ozmidov 1965), for which the horizontal Froude number $F_{h}\left(l_{o}\right)=u\left(l_{o}\right) /\left(N l_{o}\right)$ is of order unity, where $u\left(l_{o}\right)=\varepsilon_{K}{ }^{1 / 3} l_{o}^{-1 / 3}$ is the characteristic velocity associated with the length scale $l_{o}$.

However, numerous numerical simulations of stratified turbulence report mixing events due to the shear instability (Laval et al. 2003; Riley \& de Bruyn Kops 2003; Hebert \& de Bruyn Kops 2006; Brethouwer et al. 2007; Waite 2011). As shown by Riley \& de Bruyn Kops (2003), the inverse of the buoyancy Reynolds number is an estimate of the minimum value of the Richardson number that can be reached when vertical diffusion balances horizontal transport. Thus, the condition $\mathscr{R}>1$ can be interpreted as a condition for the development of the shear instability in stratified turbulence. In addition, the Richardson number is related to the vertical Froude number $R i \sim\left(N L_{v} / U\right)^{2} \sim 1 / F_{v}{ }^{2}$ so that overturnings might develop at vertical length scales $L_{v}$ of the order of the buoyancy length scale $L_{b}$, i.e. at scales larger than the Ozmidov length scale $l_{o}$.

The evolution of a counter-rotating vortex pair in a stratified fluid has been extensively studied, in particular because it is one of the simplest flows on which the zigzag instability develops and from which the buoyancy length scale naturally emerges as the vertical length (Billant \& Chomaz 2000a,b,c; Otheguy, Chomaz \& Billant 2006; Billant 2010; Billant et al. 2010). Recently, Deloncle, Billant \& Chomaz (2008), Waite \& Smolarkiewicz (2008) and Augier \& Billant (2011) have investigated the nonlinear development of the zigzag instability. They have shown that both the shear and gravitational instabilities appear at high buoyancy Reynolds number when the zigzag instability has a finite amplitude leading to a transition to turbulence. This simple flow is of interest to unfold the nonlinear processes and instabilities 
since they occur successively in time, whereas in stratified turbulence they all operate simultaneously.

In this paper, we continue the numerical study of the transition to turbulence in this particular case of a dipole. In contrast to previous studies, we focus on the spectral properties and transfers. In $\S 2$, we describe the initial conditions and the numerical methods. The evolution of the spectra for a moderate horizontal Froude number is described in detail in $\S 3$. The effect of the horizontal Froude number is studied in $\S 4$ and that of the Reynolds number in $\S 5$. A spectral analysis of the nonlinear transfers is presented in $\S 6$. Finally, conclusions are offered in the last section.

\section{Methods}

The governing equations are the incompressible Navier-Stokes equations under the Boussinesq approximation with hyperviscosity:

$$
\begin{aligned}
\partial_{t} \boldsymbol{u}+\boldsymbol{u} \cdot \nabla \boldsymbol{u} & =-\frac{1}{\rho_{0}} \nabla p-\frac{g \rho^{\prime}}{\rho_{0}} \boldsymbol{e}_{z}+\left(v \nabla^{2}+v_{4} \nabla^{8}\right) \boldsymbol{u}, \\
\partial_{t} \rho^{\prime}+\boldsymbol{u} \cdot \nabla \rho^{\prime} & =N^{2} u_{z}+\left(\kappa \nabla^{2}+v_{4} \nabla^{8}\right) \rho^{\prime},
\end{aligned}
$$

where $\boldsymbol{u}=\left(u_{x}, u_{y}, u_{z}\right)$ is the non-divergent velocity $(\boldsymbol{\nabla} \cdot \boldsymbol{u}=0)$ in Cartesian coordinates $(x, y, z)$ with $z$ the vertical coordinate, $p$ is the pressure, $\boldsymbol{e}_{z}$ is the vertical unit vector, $g$ is the acceleration due to gravity, $\rho^{\prime}(x, y, z, t)$ is the perturbation density relative to the sum of a constant reference density $\rho_{0}$ and a linear density profile $\bar{\rho}(z), N=\sqrt{-\left(g / \rho_{0}\right)(\mathrm{d} \bar{\rho} / \mathrm{d} z)}$ is the constant Brunt-Väisälä frequency, $v$ the kinematic viscosity, $\kappa$ the mass diffusivity and $v_{4}$ is a constant hyperviscosity coefficient added for numerical purpose (see below).

The methods are similar to those employed in Augier \& Billant (2011). The numerical simulations are initialized by a Lamb-Chaplygin columnar dipole weakly perturbed by the dominant mode of the zigzag instability (for the analytic expression of the initial state, see Augier \& Billant 2011). Equations (2.1)-(2.2) are solved by means of a pseudo-spectral method with periodic boundary conditions (see Deloncle et al. 2008 for details). Time advancement is carried out with the classical fourth-order Runge-Kutta scheme for the nonlinear term and exact integration for the viscous and diffusive terms. Most of the aliasing is removed by truncating 9/10 of the modes along each direction.

The Reynolds number $R e$ and the horizontal Froude number $F_{h}$ are based on the initial conditions: $R e=U R / v, F_{h}=U /(N R)$, where $U$ and $R$ are respectively the velocity of translation and the radius of the Lamb-Chaplygin dipole. The Schmidt number $S c=v / \kappa$ is set to unity in all runs. For simplicity and without loss of generality, $R$ and $R / U$ are taken respectively as length and time units. The density perturbations are non-dimensionalized by $R \mathrm{~d} \bar{\rho} / \mathrm{d} z$. The vertical length of the numerical box $\mathscr{L}_{z}$ is taken equal to the vertical wavelength of the dominant mode of the zigzag instability $\lambda_{z z} / R \simeq 10 F_{h}$ (Billant \& Chomaz 2000c). Throughout the paper, the buoyancy length scale will be set to this length scale $L_{b}=\lambda_{z z}=10 U / N$.

Most of the simulations are performed for the same Reynolds number: $R e=28000$. In contrast, the Froude number is varied from $F_{h}=0.0225$ (strong stratification) to $F_{h}=0.135$ (moderate stratification). Thus a large range of buoyancy Reynolds number is covered going from 14 to 510, i.e. always well above the threshold for the shear and gravitational instabilities $\mathscr{R}_{c} \simeq 4$.1 for the Lamb-Chaplygin dipole (Augier \& Billant 2011). The parameters of the runs are summarized in table 1. 


\begin{tabular}{|c|c|c|c|c|c|c|c|c|c|}
\hline Run & $F_{h}$ & $\frac{R e}{1000}$ & $\mathscr{R}$ & $\mathscr{L}_{z}$ & $N_{h}^{2} \times N_{z}$ & $1 / \operatorname{Re}_{4}$ & $\max _{t}\left(\frac{\varepsilon_{v_{4}}(t)}{\varepsilon(t)}\right)$ & $\max _{t}\left(\varepsilon_{K}(t)\right)$ & $k_{\max } \eta$ \\
\hline R1S & 0.0225 & 28 & 14 & 0.225 & $384^{2} \times 24$ & $1.8 \times 10^{-18}$ & 0.63 & 0.088 & 0.26 \\
\hline R1M & 0.0225 & 28 & 14 & 0.225 & $768^{2} \times 48$ & $9.3 \times 10^{-21}$ & 0.40 & 0.082 & 0.52 \\
\hline $\mathrm{R} 1 \mathrm{~L}$ & 0.0225 & 28 & 14 & 0.225 & $1024^{2} \times 64$ & $1.1 \times 10^{-21}$ & 0.31 & 0.082 & 0.69 \\
\hline $\mathrm{R} 2 \mathrm{~S}$ & 0.045 & 28 & 57 & 0.45 & $384^{2} \times 48$ & $1.8 \times 10^{-18}$ & 0.71 & 0.075 & 0.27 \\
\hline $\mathrm{R} 2 \mathrm{M}$ & 0.045 & 28 & 57 & 0.45 & $768^{2} \times 96$ & $9.3 \times 10^{-21}$ & 0.50 & 0.088 & 0.51 \\
\hline $\mathrm{R} 2 \mathrm{~L}$ & 0.045 & 28 & 57 & 0.45 & $1024^{2} \times 128$ & $1.1 \times 10^{-21}$ & 0.38 & 0.087 & 0.68 \\
\hline R3S & 0.09 & 28 & 227 & 0.9 & $384^{2} \times 96$ & $1.8 \times 10^{-18}$ & 0.73 & 0.066 & 0.27 \\
\hline R3T1 & 0.09 & 7 & 57 & 0.9 & $768^{2} \times 192$ & 0 & 0 & 0.062 & 1.58 \\
\hline R3T2 & 0.09 & 14 & 113 & 0.9 & $768^{2} \times 192$ & $8.9 \times 10^{-21}$ & 0.23 & 0.066 & 0.92 \\
\hline $\mathrm{R} 3 \mathrm{M}$ & 0.09 & 28 & 227 & 0.9 & $768^{2} \times 192$ & $9.3 \times 10^{-21}$ & 0.49 & 0.070 & 0.54 \\
\hline R3L & 0.09 & 28 & 227 & 0.9 & $1024^{2} \times 256$ & $1.1 \times 10^{-21}$ & 0.36 & 0.069 & 0.72 \\
\hline R3T3 & 0.09 & 28 & 227 & 0.9 & $1280^{2} \times 320$ & $1.7 \times 10^{-22}$ & 0.25 & 0.069 & 0.91 \\
\hline R4S & 0.135 & 28 & 510 & 1.35 & $384^{2} \times 144$ & $1.8 \times 10^{-18}$ & 0.73 & 0.063 & 0.28 \\
\hline $\mathrm{R} 4 \mathrm{M}$ & 0.135 & 28 & 510 & 1.35 & $768^{2} \times 288$ & $9.3 \times 10^{-21}$ & 0.49 & 0.063 & 0.56 \\
\hline R4L & 0.135 & 28 & 510 & 1.35 & $1024^{2} \times 384$ & $1.1 \times 10^{-21}$ & 0.36 & 0.062 & 0.74 \\
\hline
\end{tabular}

TABLE 1. Overview of the physical and numerical parameters of the simulations. For all simulations $\mathscr{L}_{h}=4$. The number of nodes in the $x$-, $y$ - and $z$-direction are denoted $N_{x}, N_{y}$ and $N_{z}$, respectively, with $N_{x}=N_{y}=N_{h}$. We recall that the length and time units are $R$ and $R / U$, respectively; $\varepsilon_{v_{4}}(t), \varepsilon(t)$ and $\varepsilon_{K}(t)$ denote the hyperviscous dissipation, the total dissipation and the dissipation of kinetic energy, respectively; $\eta=\left[v^{3} / \max \left(\varepsilon_{K}(t)\right)\right]^{1 / 4}$ is the Kolmogorov length scale and $k_{\max }$ the maximum wavenumber.

In order to achieve such a high Reynolds number, our methods differ from those employed in Augier \& Billant (2011) in four main points. First, we use an adaptable time step procedure which maximizes the time step over a Courant-Friedrichs-Lewy condition (Lundbladh et al. 1999; Augier 2011).

Second, the horizontal size of the box is $\mathscr{L}_{h}=4$ instead of $\mathscr{L}_{h}=10$. We have verified that the development of the zigzag, shear and gravitational instabilities are not significantly affected by this stronger lateral confinement due to the periodic boundary conditions. As shown in the Appendix, the shape of the horizontal kinetic energy spectra is almost independent of the horizontal size of the box. This indicates that the dynamics and the size of the turbulent structures generated by the different instabilities do not depend on the horizontal size of the box. Only the spectra at the largest scales vary and the late evolution differs when the pancake dipoles issued from the zigzag instability have travelled more than the horizontal size of the box and the amplitude of the zigzag instability is larger than the box size.

Third, in order to reduce the computational cost, the resolution in the $x$ - and $y$-directions is increased during the run so as to adapt to the smallest scales of the flow. We start with a horizontal resolution $N_{x} \times N_{y}=384 \times 384$. When the zigzag instability becomes nonlinear $(t=3)$, the resolution is increased to $768 \times 768$, and when the secondary instabilities develop $(t=3.7)$, it is set to $1024 \times 1024$. The runs for these 
three different resolutions are labelled $\mathrm{S}, \mathrm{M}$ and $\mathrm{L}$ respectively (table 1). For each horizontal resolution, the number of numerical points in the vertical direction, $N_{z}$, is chosen so as to have a nearly isotropic mesh $N_{z} \simeq\left(\mathscr{L}_{z} / \mathscr{L}_{h}\right) N_{x}$.

Fourth, to ensure the numerical stability, we have added to the classical dissipation an isotropic hyperviscosity (see (2.1)-(2.2)). The value of the hyperviscosity $1 / R e_{4}=$ $v_{4} /\left(U R^{7}\right)$ is decreased when the resolution is increased such that only the modes with the highest wavenumbers significantly contribute to the hyperviscous dissipation. More precisely, in order to choose the hyperviscosity coefficient $v_{4}$, we have first performed a series of direct numerical simulation (DNS) in order to determine the required viscosity $v_{D N S}$ as a function of the maximum wavenumber $k_{\max }$. The results have then been fitted with a law derived from the Kolmogorov length scale $v_{D N S}\left(k_{\max }\right)=\check{\epsilon}^{1 / 3} k_{\max }^{-4 / 3}$, where the sole parameter $\check{\epsilon}$ is obtained from the fit. The hyperviscosity coefficient has then been determined by requiring that the sum of the normal and hyper- dissipations is equal to the dissipation for an equivalent DNS extrapolated from the previous law $v\left(\alpha k_{\max }\right)^{2}+v_{4}\left(\alpha k_{\max }\right)^{8}=v_{\mathrm{DNS}}\left(k_{\max }\right) k_{\max }{ }^{2}$, where $\alpha$ is a parameter set to 0.85 such that the peak in the dissipation spectra is resolved. For each resolution, the hyperdissipation rate $\varepsilon_{v_{4}}(t)$ is observed to suddenly increase at a particular time when the highest wavenumber modes start to be filled. The resolution is increased before this time, i.e. before the hyperviscosity affects the flow (except for the highest resolution).

The lack of resolution can be quantified by the temporal maximum (which is at the maximum of dissipation) of the ratio $\varepsilon_{v_{4}}(t) / \varepsilon(t)$, where $\varepsilon(t)$ is the total energy dissipation rate. This quantity would tend to 0 if the smallest resolved scales were much smaller than the Kolmogorov length scale $\eta$. In table 1, the value of this parameter is reported for each run (note that all simulations are continued long after the maximum of dissipation). It is around $0.3-0.4$ for the simulations with $N_{h}=1024$.

Table 1 also indicates the product $k_{\max } \eta$, where $k_{\max }$ is the maximum wavenumber and the Kolmogorov length scale is defined as $\eta=\left[v^{3} / \max \left(\varepsilon_{K}(t)\right)\right]^{1 / 4}$ since the turbulence invades nearly the whole numerical domain during the period of maximum dissipation. This quantity varies between 0.3 and 0.9 , thus being close to unity as required in DNS (de Bruyn Kops \& Riley 1998; Brethouwer et al. 2007).

In order to investigate the effect of the hyperviscosity, we have carried out three additional simulations for $F_{h}=0.09$ : simulations R3T1 and R3T2 with the same resolution $N_{h}{ }^{2} \times N_{z}=768^{2} \times 192$ as simulation R3M but a different Reynolds number, $R e=7000$ and $R e=14000$ respectively, and simulation R3T3 with a larger resolution $N_{h}{ }^{2} \times N_{z}=1280^{2} \times 320$ than simulation R3L but the same Reynolds number $R e=28000$. The simulation R3T1 is a DNS with $v_{4}=0$ and $k_{\max } \eta=1.58$ and simulations R3T2 and R3T3 are very close to DNS with $k_{\max } \eta \simeq 0.9$. Some results obtained from these simulations are presented and discussed in $\S 5$.

A careful comparison of runs R3L and R3T3 corresponding to the same set of parameters but to two slightly different resolutions does not show any differences in physical and spectral space apart from the width of the dissipative range. Indeed, the maximum of the ratio $\varepsilon_{v_{4}}(t) / \varepsilon(t)$ has decreased from 0.36 to 0.25 . Even though the two resolutions are not very different, this suggests that, when the resolution is large enough, the non-dissipative part of the flow starts to be independent of the resolution and of the associated hyperviscosity suggesting that it would also be the same if a DNS were carried out. Because we do not seek to study the detailed structure of the dissipative range provided that both the buoyancy and the Ozmidov scales are well resolved, such hyperviscosity allows us to decrease the width of the dissipation range 
and achieve higher Reynolds number values, i.e. increase the width of the inertial range for a given computational cost.

\section{Global description of a simulation with $F_{h}=0.09$}

As already described by Deloncle et al. (2008), Waite \& Smolarkiewicz (2008) and Augier \& Billant (2011), the zigzag instability develops linearly at the beginning of the simulation and bends the dipole. By $t=3.3$, the amplitude of the bending deformations is large but no secondary instability is active yet (Augier \& Billant 2011). Thus, we begin our description of the flow at $t=3.3$.

Figure $1(a, d, g, j)$ presents the time evolution of the density field for $F_{h}=0.09$ in a horizontal cross-section at the level at which the shear instability appears, $z=0.66 \mathscr{L}_{z}$. At $t=3.8$, small-scale wiggles can be seen (figure $1 d$ ). They are associated with the roll-up of Kelvin-Helmholtz $(\mathrm{KH})$ billows with an horizontal axis as described by Deloncle et al. (2008) and Augier \& Billant (2011). At time $t=4.2$ (figure $1 \mathrm{~g}$ ), the destabilization of the $\mathrm{KH}$ billows start to generate disordered small scales. Eventually, just after the maximum of dissipation, i.e. at $t=4.9$, these small scales invade a large portion of the domain (figure $1 j$ ).

To analyse the properties of these small scales, we first use the poloidal-toroidal decomposition (Cambon 2001), also known as the Craya-Herring decomposition (Craya 1958; Herring 1974), which is simply expressed in Fourier space as $\hat{\boldsymbol{u}}=\hat{\boldsymbol{u}}_{p}+\hat{\boldsymbol{u}}_{t}$ for each wavenumber, where $\hat{\boldsymbol{u}}_{p}=-\boldsymbol{e}_{\boldsymbol{\theta}} \times\left(\boldsymbol{e}_{\boldsymbol{\theta}} \times \hat{\boldsymbol{u}}\right)$ and $\hat{\boldsymbol{u}}_{t}=\left(\boldsymbol{e}_{\boldsymbol{\theta}} \cdot \hat{\boldsymbol{u}}\right) \boldsymbol{e}_{\boldsymbol{\theta}}$ with $\hat{\boldsymbol{u}}$ the velocity in Fourier space, $\boldsymbol{e}_{\boldsymbol{\theta}}$ the unit vector parallel to $\boldsymbol{e}_{z} \times \boldsymbol{k}$, where $\boldsymbol{e}_{z}$ is the vertical unit vector and $\boldsymbol{k}$ the wave vector. In the limit of small vertical Froude number, the poloidal velocity $\hat{\boldsymbol{u}}_{p}$ is associated with gravity waves and the toroidal velocity $\hat{\boldsymbol{u}}_{t}$ with potential vorticity modes. It has to be stressed that this interpretation is not legitimate here since the vertical Froude number reaches a value of order unity. Indeed, the zigzag, $\mathrm{KH}$ and Rayleigh-Taylor instabilities induce an increase of the poloidal kinetic energy $\hat{E}_{K p}(\boldsymbol{k})=\left|\hat{\boldsymbol{u}}_{p}\right|^{2} / 2$ and of the potential energy $\hat{E}_{P}(\boldsymbol{k})=\left|\hat{\rho}^{\prime}\right|^{2} /\left(2 F_{h}{ }^{2}\right)$, even though there are no waves (see e.g. Staquet \& Riley 1989). Nevertheless, the toroidal-poloidal decomposition is used here only as a convenient formal decomposition to detect the occurrence of vertical velocity without overinterpreting its meaning in term of waves and vortices. Since the poloidal velocity does not correspond to vertical vorticity, its representation allows us to follow in Fourier space both the development of the nonlinear zigzag instability with the strongly deformed dipole and the Kelvin-Helmholtz instability.

From the energy in a Fourier mode $\hat{E}_{K p}(\boldsymbol{k})$, we define a two-dimensional poloidal energy spectral density

$$
E_{K p}\left(\kappa_{h}, k_{z}\right)=\frac{1}{\delta \kappa_{h} \delta k_{z}} \sum_{\boldsymbol{k} \in \delta \Omega_{\left[\kappa_{h}, \pm k_{z}\right]}} \hat{E}_{K p}(\boldsymbol{k}),
$$

where $\delta k_{z}=2 \pi / \mathscr{L}_{z}, \delta \kappa_{h}=2 \pi / \mathscr{L}_{h}$ and $\delta \Omega_{\left[\kappa_{h}, \pm k_{z}\right]}$ is a volume made up of two annuli of thickness $\delta k_{z}$ in the vertical, located in the two planes $\pm k_{z}$ and defined horizontally by the relation $\kappa_{h}{ }^{2} \leqslant k_{x}{ }^{2}+k_{y}{ }^{2}<\left(\kappa_{h}+\delta \kappa_{h}\right)^{2}$. Figure $1(b, e, h, k)$ shows for the same instants as figure $1(a, d, g, j)$ the poloidal kinetic energy density $E_{K p}\left(\kappa_{h}, k_{z}\right)$. Both vertical and horizontal wavenumbers are scaled by $k_{b}=2 \pi / L_{b}=2 \pi / \mathscr{L}_{z}$, which corresponds to the lowest non-zero vertical wavenumber and to the most amplified wavenumber of the zigzag instability. At $t=3.3$, the poloidal kinetic energy is concentrated at $k_{z}=k_{b}$ 

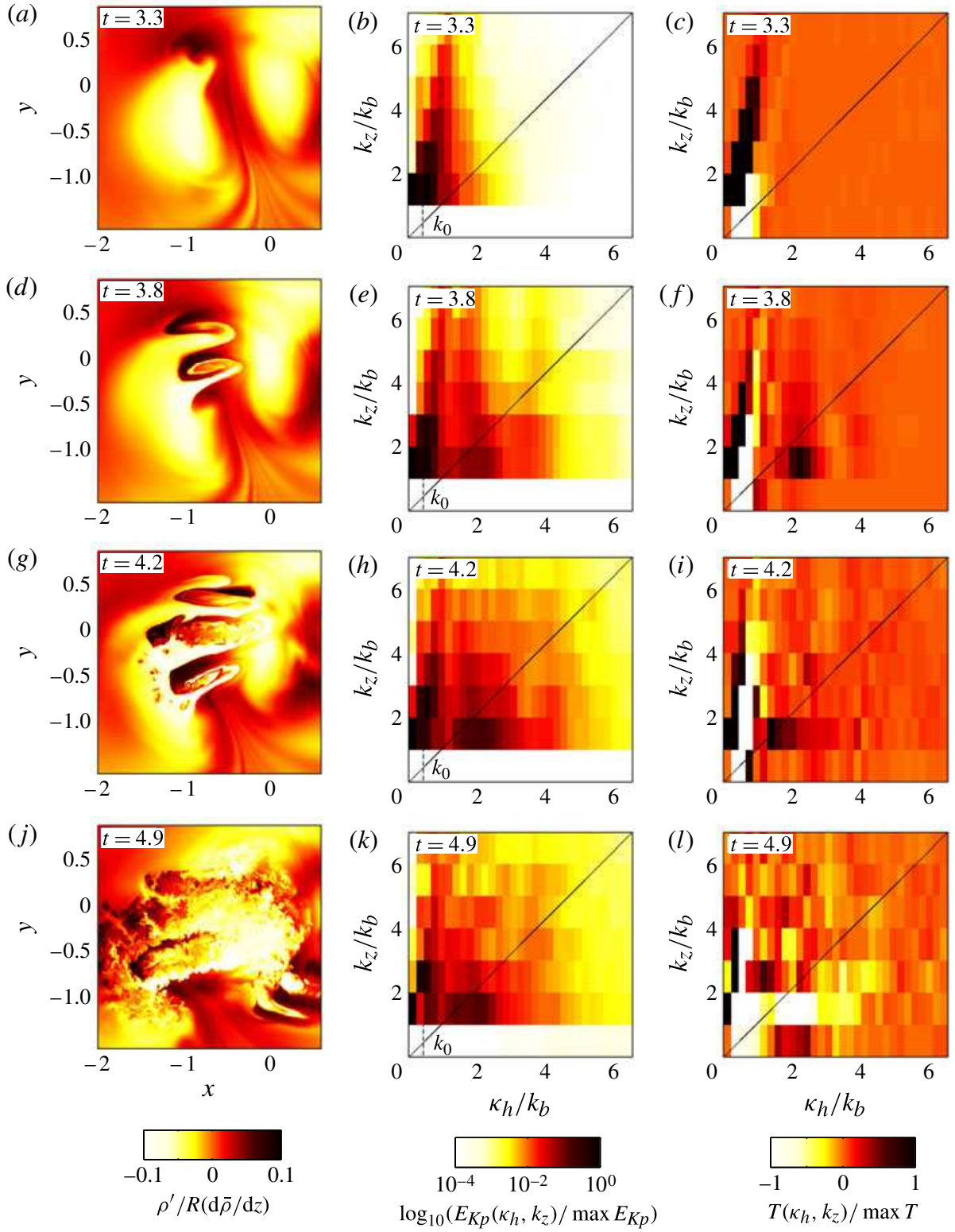

FIGURE 1. (Colour online) Description of the flow at four different characteristic times: $t=3.3(a-c), t=3.8(d-f), t=4.2(g-i)$ and $t=4.9(j-l)$ for $F_{h}=0.09 .(a, d, g, j)$ Horizontal cross-sections of the density field at the level at which the shear instability begins to develop $z=0.66 \mathscr{L}_{z} ;(b, e, h, k)$ the spectrum of poloidal energy $E_{K p}\left(\kappa_{h}, k_{z}\right)$ and $(c, f, i, l)$ the total nonlinear energy transfer $T\left(\kappa_{h}, k_{z}\right)$.

and $\kappa_{h} \simeq 0$ to $k_{0}$, where $k_{0}$ is the leading horizontal wavenumber of the twodimensional base flow (marked in figure $1 b$ ). This feature is associated with the zigzag instability that has a finite amplitude. The energy is spread out at higher vertical wavenumbers than $k_{b}$ as a result of the nonlinear development of the zigzag instability. 
Figure $1(c, f, i, l)$ shows the two-dimensional spectral density of total nonlinear energy transfers defined by the relation

$$
T\left(\kappa_{h}, k_{z}\right)=\frac{1}{\delta \kappa_{h} \delta k_{z}} \sum_{\boldsymbol{k} \in \delta \Omega_{\left[\kappa_{h}, \pm k_{z}\right]}} \hat{T}(\boldsymbol{k}),
$$

where $\hat{T}(\boldsymbol{k})=-\operatorname{Re}\left\{\hat{\boldsymbol{u}}^{*}(\boldsymbol{k}) \cdot P_{\perp}[\widehat{\boldsymbol{u} \cdot \nabla \boldsymbol{u}}](\boldsymbol{k})+F_{h}^{-2}{\hat{\rho^{\prime}}}^{*}(\boldsymbol{k})\left(\widehat{\boldsymbol{u} \cdot \nabla \rho^{\prime}}\right)(\boldsymbol{k})\right\}$, Re denoting the real part, the star the complex conjugate and $P_{\perp}$ the projection operator on the space perpendicular to $\boldsymbol{k}$ (Lindborg 2006; Waite 2011). The factor $F_{h}^{-2}$ in front of the second term comes from the non-dimensionalization of the density perturbations (see $\S 2$ ). In all the light regions, the transfer is negative meaning that these modes are losing energy to the wavenumbers in the dark regions. At $t=3.3$ (figure $1 c$ ), the loss is maximum for the two-dimensional mode $k_{z}=0, \kappa_{h}=k_{0}$ and the gain is maximum at $k_{z}=k_{b}, \kappa_{h} \simeq 0-k_{0}$. This indicates that the zigzag instability is the leading mechanism extracting energy from the two-dimensional base flow at that time. The black region extends vertically nearly up to $k_{z} \sim 6 k_{b}$ and $\kappa_{h} \simeq k_{b}$. This confirms that the nonlinear development of the zigzag instability before the secondary instability is transferring energy to vertical harmonic modes of the initial preferred vertical wavenumber $k_{b}$, i.e. to small vertical scales but not to horizontal scales smaller than the buoyancy length scale. Figure 1(c) also shows transfer toward the modes $k_{z}=k_{b}$ and $\kappa_{h}=0$ which are the so-called 'shear modes' reported by Smith \& Waleffe (2002).

At $t=3.8$, the energy transfer (figure $1 f$ ) exhibits a new peak close to $k_{z} \simeq k_{b}$ and $\kappa_{h} \simeq 2 k_{b}$ (just below the diagonal isotropic line $\kappa_{h}=k_{z}$ ), due to the appearance of the $\mathrm{KH}$ instability, which starts when the local Richardson number is small enough $R i \lesssim 1 / 4$ (Deloncle et al. 2008). The wavenumber selected by the KH instability scales like the shear thickness, which is proportional to the buoyancy length $L_{b}$ when $R i$ is close to the critical value for instability. Therefore, the horizontal wavenumber selected by the secondary $\mathrm{KH}$ instability scales like $k_{b}$. The poloidal kinetic energy (figure 1e) presents at that time a secondary peak around $\kappa_{h} \simeq 2 k_{b}$ just below the 'isotropic' diagonal $\left(\kappa_{h}=k_{z}\right)$. At $t=4.2$, the appearance of small scales due to the destabilization of the $\mathrm{KH}$ billows (figure $1 \mathrm{~g}$ ), corresponds to positive transfers (figure $1 i$ ) toward high horizontal wavenumbers and a loss of energy at low horizontal wavenumbers. The poloidal kinetic energy spectrum exhibits a more isotropic shape with energy distributed nearly uniformly along the semicircular lines $k_{z}^{2}+\kappa_{h}^{2}=$ const (figure $1 h$ ). At $t=4.9$ (figure $1 l$ ), there are eventually transfers toward very small scales and all the scales corresponding to the earlier development of the $\mathrm{KH}$ billows are now losing energy (bright region). It can be noticed that during this whole process, the modes $k_{z} \simeq k_{b}$ to $3 k_{b}$ and $\kappa_{h} \simeq k_{0}$ are still gaining energy indicating that the primary zigzag instability remains active despite the development of the secondary shear and gravitational instabilities.

In figure 2, we have plotted several horizontal (black curves) and vertical (light curves) instantaneous compensated one-dimensional spectra $E_{K}\left(k_{h}\right) \varepsilon_{K}^{-2 / 3} k_{h}^{5 / 3}$ and $E_{K}\left(k_{z}\right) \varepsilon_{K}^{-2 / 3} k_{z}^{5 / 3}$, respectively, for the time $3.8 \leqslant t \leqslant 5.2$ corresponding to the development and the destabilization of the KH billows. The inset plot also shows the spectra for $t \leqslant 3.8$, i.e. prior to the development of the shear instability. The sloping straight lines indicate the $k^{-3}$ power law and the horizontal lines display the $C \varepsilon_{K}^{2 / 3} k^{-5 / 3}$ law, with $C=0.5$. Here $\varepsilon_{K}$ is the maximum kinetic energy dissipation rate and the horizontal one-dimensional spectra are computed in the same way as in Lindborg 


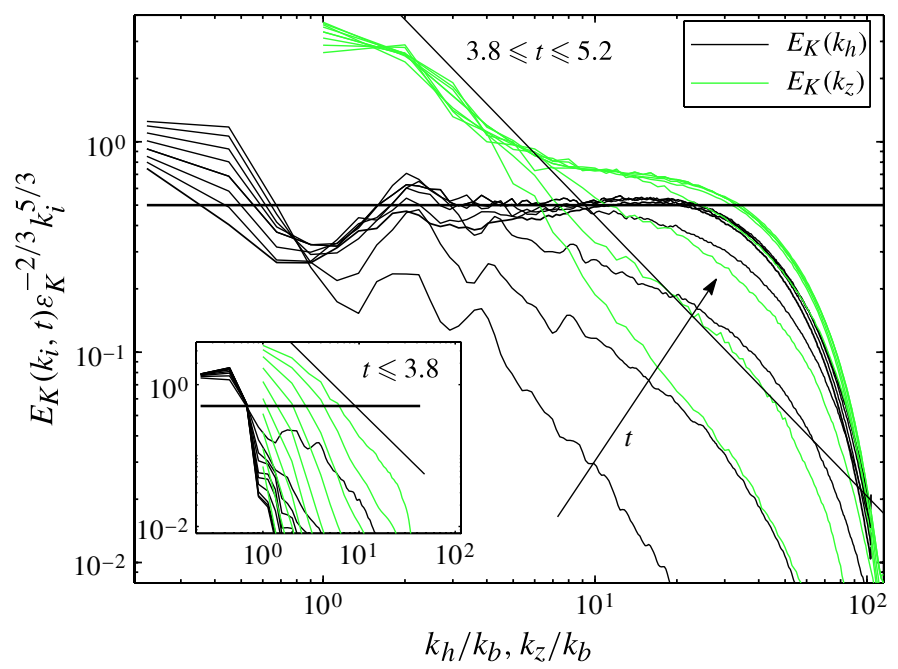

FIgURE 2. (Colour online) Time evolution of the horizontal and vertical compensated onedimensional spectra for $F_{h}=0.09$ and $R e=28000$. In the main plot, the spectra are shown for $t=3.8-5.2$ with a time increment equal to 0.2 . The inset plot shows the spectra for $t=0.2-3.8$ with a time increment of 0.4 . The thick curves correspond to the time for which the dissipation is maximum. The sloping straight lines indicate the $k_{z}^{-3}$ power law and the horizontal lines the $C \varepsilon_{K}^{2 / 3} k^{-5 / 3}$ law, with $C=0.5$.

(2006) as the mean value of the $k_{x}$ and the $k_{y}$ spectra,

$$
E_{K}\left(k_{h}\right)=\frac{1}{2 \delta k_{h}}\left[\sum_{\substack{k_{h} \leqslant\left|k_{x}\right|<k_{h}+\delta k_{h} \\ k_{y}, k_{z}}} \hat{E}_{K}(\boldsymbol{k})+\sum_{\substack{k_{h} \leqslant\left|k_{y}\right|<k_{h}+\delta k_{h} \\ k_{x}, k_{z}}} \hat{E}_{K}(\boldsymbol{k})\right],
$$

where $\delta k_{h}=2 \pi / \mathscr{L}_{h}$ and $\hat{E}_{K}(\boldsymbol{k})=|\hat{\boldsymbol{u}}|^{2} / 2$. Note the difference between the horizontal wavenumber $k_{h}$ and the modulus of the horizontal wave vector $\kappa_{h}$. The use of the symbol $k_{h}$ indicates that the spectral quantities considered are the average of the onedimensional spectra in the $x$ - and $y$-directions, meaning that the volumes of integration in the Fourier space are parallelepipeds as indicated by the summation in (3.3). These spectra are very similar to spectra obtained with one-dimensional measurements as for example in Nastrom \& Gage (1985). Moreover, they can be directly compared with vertical one-dimensional spectra, functions of the vertical wavenumber $k_{z}$. In contrast, the use of the symbol $\kappa_{h}$ indicates that the spectral quantities considered are two-dimensional spectra, integrated isotropically on the horizontal, the volume of integration being annuli as indicated by the summation in (3.1). The two-dimensional spectra are fully adapted for the Craya-Herring decomposition (for a more precise discussion on the differences between one- and two-dimensional spectra, see Lindborg \& Brethouwer 2007).

In the inset plot, we see that during the early evolution of the zigzag instability, the horizontal spectrum does not vary. Only after $t>3.2$ does the energy at horizontal wavenumbers larger than the buoyancy wavenumber $k_{b}$ start increasing. In sharp contrast, the level of the vertical spectra increases nearly linearly in time in this 

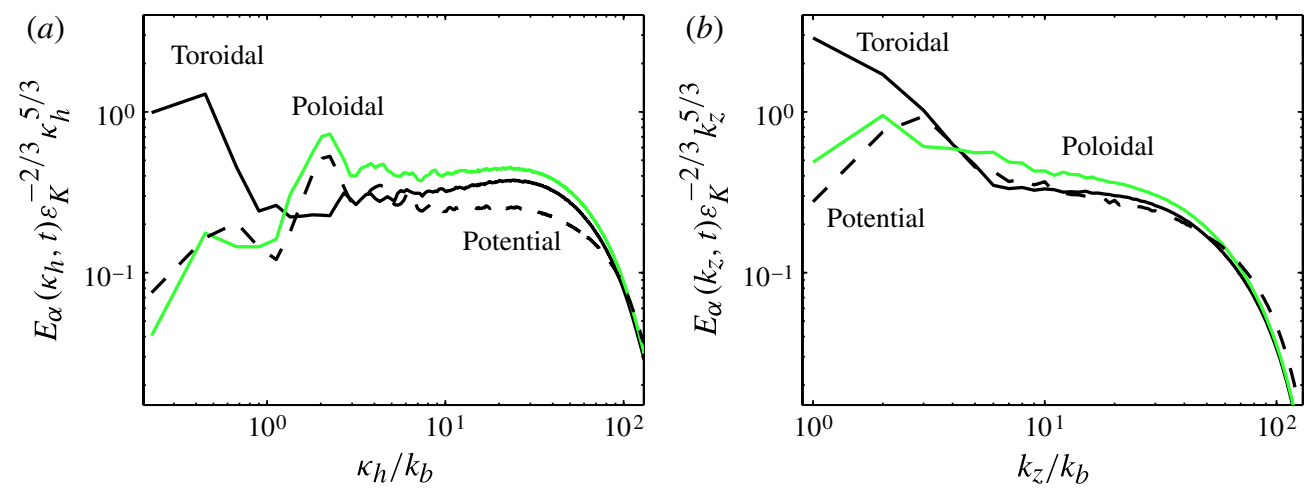

FIGURE 3. (Colour online) Horizontal two-dimensional $(a)$ and vertical one-dimensional $(b)$ compensated spectra of toroidal, poloidal and potential energy for $F_{h}=0.09$ and $R e=28000$ at $t=4.6$. Black, light and dashed curves correspond respectively to toroidal, poloidal and potential energies.

logarithmic representation since the energy in the first mode grows exponentially owing to the development of the zigzag instability. For the penultimate time of the inset, $t=3.4$, the slope of the vertical spectra approaches $k_{z}^{-3}$ even though only the zigzag instability has developed at that time. This indicates that this characteristic slope is mainly due to the vertical deformations of the dipole induced by the zigzag instability.

Starting at $t=3.4$, we can see a peak in the horizontal spectra around $k_{h} / k_{b}=2$ and its harmonics at $k_{h} / k_{b}=4$ and 8 . This is due to the appearance of the secondary $\mathrm{KH}$ instability. We observe a dip in the horizontal spectra between the small horizontal wavenumber $k_{0}$, corresponding to the initial dipole, and $k_{h} / k_{b}=2$. This is consistent with the observation of figure 1 where a direct transfer from the $\left[\kappa_{h}, k_{z}\right] \simeq\left[k_{0}, k_{b}\right]$ modes to the $\left[\kappa_{h}, k_{z}\right] \simeq\left[2 k_{b}, k_{b}\right]$ modes due to the $\mathrm{KH}$ instability was demonstrated. Beyond $t=4.4$, energy eventually cascades toward the small horizontal scales with a slope close to $k_{h}^{-5 / 3}$ over approximately one decade. Remarkably, the horizontal spectra nearly perfectly collapse onto the $C \varepsilon_{K}{ }^{2 / 3} k_{h}^{-5 / 3}$ law line, with $C=0.5$, as observed in forced strongly stratified turbulence (Lindborg 2006; Brethouwer et al. 2007). However, the particular value of this constant is not meaningful here since it depends on the horizontal size of the computational domain compared to the dipole size. This is because the turbulence is concentrated around the vortices and is not homogeneous along the horizontal directions. Therefore, the observed agreement with the theory of strongly stratified turbulence is fortuitous. Quite remarkably, small vertical scales develop at the same time as the horizontal scales and, after $t=4.4$, the vertical spectra exhibit a break in the slope around $k_{z} / k_{b}=8$ where the slope goes from $k_{z}^{-3}$ to nearly $k_{z}^{-5 / 3}$. The $k_{z}^{-5 / 3}$ power law begins when the vertical spectrum $k_{z}^{-3}$ approaches the horizontal one, indicating a return to isotropy.

In figure 3, instantaneous toroidal (black line), poloidal (light line) and potential (dashed line), horizontal two-dimensional (figure $3 a$ ) and vertical one-dimensional (figure $3 b$ ) spectra are presented for $t=4.6$ which corresponds to the time at which the dissipation is maximum. The large horizontal scales are dominated by the toroidal component while the peak at horizontal scales $\kappa_{h}=2 k_{b}$ is largely dominated by the poloidal and the potential components. At smaller horizontal scales, the toroidal and poloidal spectra approach each other. In figure $3(b)$, we see that the large vertical 


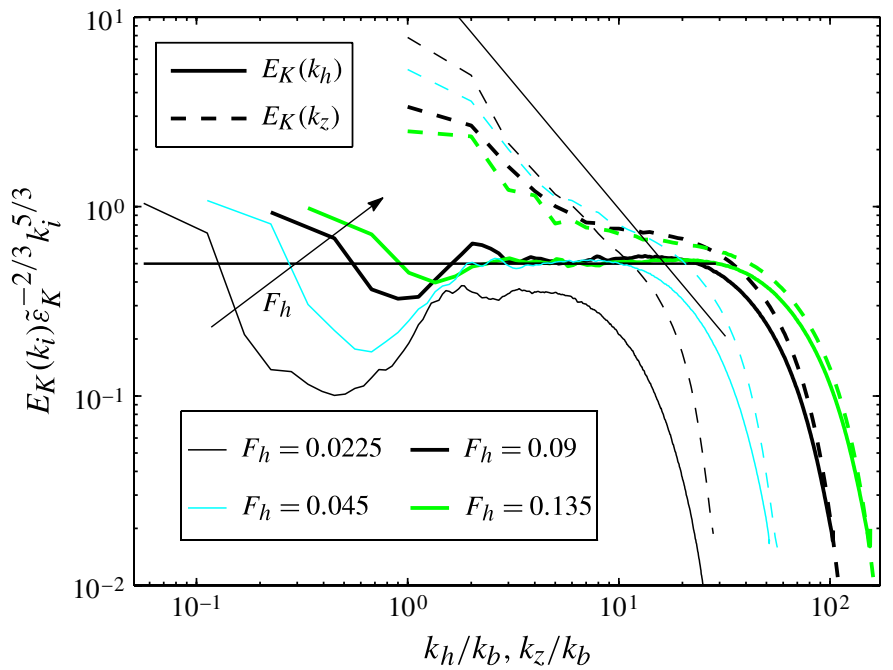

FIGURE 4. (Colour online) Horizontal (continuous curves) and vertical (dashed) kinetic energy compensated one-dimensional spectra $E_{K}\left(k_{i}\right) \tilde{\varepsilon}_{K}^{-2 / 3} k_{i}^{5 / 3}$ as a function of the dimensionless wavenumber $k_{i} / k_{b}$ for four runs with different values of the Froude number $F_{h}=0.0025,0.045,0.09$ and 0.135 but the same Reynolds number $R e=28000$. Each curve is the average over time interval $\Delta t=0.3$ near the maximum of the dissipation. The sloping straight line indicates the $k_{z}^{-3}$ power law and the horizontal line the $C \tilde{\varepsilon}_{K}^{2 / 3} k^{-5 / 3}$ law, with $C=0.5$.

scales are also dominated by the toroidal spectra, which confirms that the slope close to $k_{z}^{-3}$ in figure 2 is due to the nonlinear vertical deformations of the dipole generated by the zigzag instability.

\section{Variation of $F_{h}$ and $\mathscr{R}$}

Figure 4 presents horizontal (continuous curves) and vertical (dashed curves) compensated kinetic spectra $E_{K}\left(k_{h}\right) \tilde{\varepsilon}_{K}^{2 / 3} k_{h}^{5 / 3}$ and $E_{K}\left(k_{z}\right) \tilde{\varepsilon}_{K}^{2 / 3} k_{z}^{5 / 3}$ obtained from simulations with different values of $F_{h}$ but the same Reynolds number $R e=28000$. The spectra have been time-averaged over $\Delta t=0.3$ around the time where the total dissipation is maximum. In order to rescale quantities, we use the maximum kinetic dissipation rate for $F_{h}=0.09: \tilde{\varepsilon}_{K}=0.069$. As seen in table 1, $\varepsilon_{K}$ varies only weakly with $F_{h}$. This is because $\varepsilon_{K}$ can be considered as the energy injection rate, which is independent of $F_{h}$ since the initial state is identical for all simulations. In any case, the plot would have been very similar if each curve were scaled by its maximum dissipation rate $\varepsilon_{K}$.

All the vertical spectra begin at the same dimensionless wavenumber $k_{z} / k_{b}=1$ because the vertical size of the numerical box is adjusted to the dominant wavelength of the zigzag instability. While $R e$ is the same for all runs, the dissipative ranges extend to larger values of $k_{h} / k_{b}$ when $F_{h}$ is increased because $k_{b}$ decreases. For the same reason, the horizontal spectra also move to the right when $F_{h}$ increases since the lowest horizontal wavenumber is the same for all the simulations. For all the Froude numbers, the spectra are depleted in energy between the small horizontal wavenumbers and $k_{h}=k_{b}$. Remarkably, for higher wavenumbers $k_{h} \gtrsim 2 k_{b}$ all the runs except for $F_{h}=0.0225$ present a flat compensated horizontal spectrum (corresponding to a $k_{h}^{-5 / 3}$ 

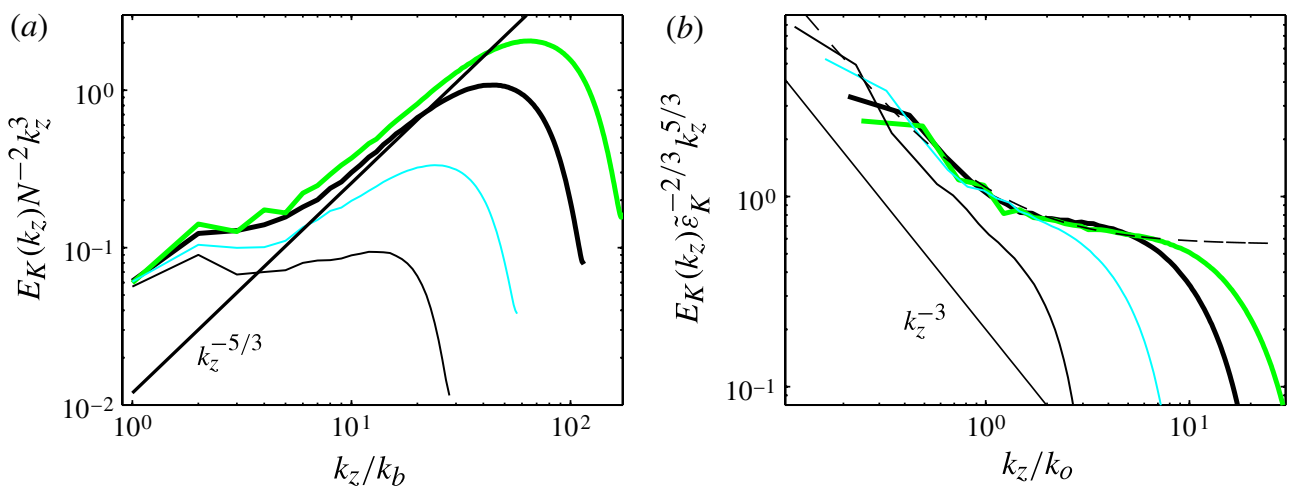

FIGURE 5. (Colour online) Vertical spectra already presented in figure 4 but plotted in $(a)$ as $E_{K}\left(k_{z}\right) N^{-2} k_{z}^{3}$ versus $k_{z} / k_{b}$ and in $(b)$ as $E_{K}\left(k_{z}\right) \tilde{\varepsilon}_{K}^{-2 / 3} k_{z}^{5 / 3}$ versus $k_{z} / k_{o}$. In $(a)$, the thick straight line indicates the $k_{z}^{-5 / 3}$ power law. In $(b)$, the thin straight line indicates the $k_{z}^{-3}$ power law and the dashed line represents the theoretical expression (4.1).

power law) collapsing at a value approximately equal to 0.5 . For $F_{h}=0.0225$ (black continuous thin line), the constant is lower, probably because of the too low value of the buoyancy Reynolds number $(\mathscr{R}=14)$.

The vertical spectra are very steep near $k_{z}=k_{b}$ and show a tendency to follow a $k_{z}^{-3}$ slope. They flatten when approaching the horizontal spectra at large wavenumbers and their slope tends to $k_{z}^{-5 / 3}$ except for the highest stratification $F_{h}=0.0025$, where the two curves approach each other only in the dissipation range (i.e. the Ozmidov scale is of the order of the Kolmogorov scale).

Figure 5(a) presents the same vertical spectra but now compensated by $N^{2} k_{z}^{-3}$, i.e. $E_{K}\left(k_{z}\right) N^{-2} k_{z}{ }^{3}$. All the curves collapse to $E_{K}\left(k_{z}\right) N^{-2} k_{z}{ }^{3}=C_{N} \simeq 0.1$ for $k_{z}=k_{b}$ corresponding to the dominant mode of the zigzag instability. However, the curves depart rapidly from this constant when $k_{z}$ increases, more so when $F_{h}$ is large. This is because the transition to the $k_{z}^{-5 / 3}$ power law occurs at a lower vertical wavenumber when $F_{h}$ increases.

Spectra scaling like $N^{2} k_{z}^{-3}$ are widely observed in nature (see e.g. Garrett \& Munk 1979; Gregg 1987) and many authors (e.g. Lumley 1964; Holloway 1983; Dewan 1997; Brethouwer et al. 2007; Riley \& Lindborg 2008) have predicted with dimensional analysis based on different theories that this spectrum should be followed by an $\varepsilon_{K}^{2 / 3} k_{z}^{-5 / 3}$ spectrum at small scales. Following Lumley (1964), we model the total spectrum as the sum of a strongly stratified spectrum and an inertial spectrum, i.e. as

$$
E_{K}\left(k_{z}\right)=C_{N} N^{2} k_{z}^{-3}+C \varepsilon_{K}^{2 / 3} k_{z}^{-5 / 3}=\left(\left(k_{z} / k_{o}\right)^{-4 / 3}+1\right) C \varepsilon_{K}^{2 / 3} k_{z}^{-5 / 3},
$$

where $C$ is a constant of order unity and $k_{o}=2 \pi / l_{o}$ with $l_{o}=2 \pi\left(C / C_{N}\right)^{3 / 4}\left(\varepsilon_{K} / N^{3}\right)^{1 / 2}$ the Ozmidov length scale. In figure $5(b)$, the compensated vertical spectra $E_{K}\left(k_{z}\right) \tilde{\varepsilon}_{K}^{-2 / 3} k_{z}^{5 / 3}$ are plotted as a function of $k_{z} / k_{o}$. Except for the Froude number $F_{h}=0.0225$, all the curves collapse over a large range of vertical wavenumbers and in particular near the wavenumber of transition between the $k_{z}^{-3}$ and the $k_{z}^{-5 / 3}$ power laws. This indicates that the change of slope occurs at the Ozmidov length scale as predicted for strongly stratified turbulence (Brethouwer et al. 2007). We can see that the spectrum (4.1) with $C_{N}=0.08$ and $C=0.56$ (dashed line) describes remarkably well the observed spectra, except of course near the dissipative range. It 

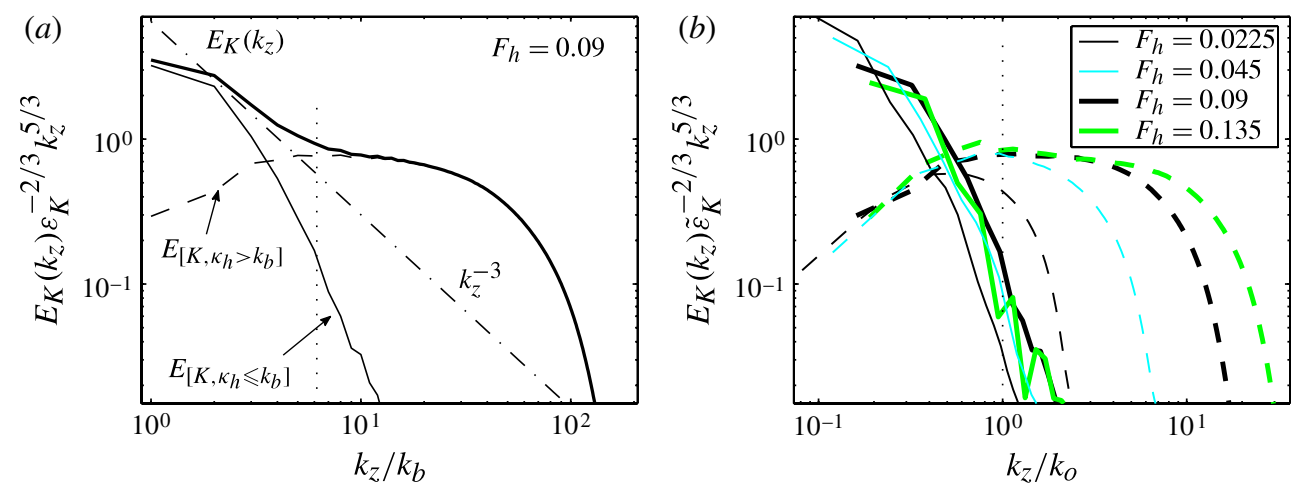

FigURE 6. (Colour online) Decomposition of the vertical compensated spectra for $F_{h}=0.09$ $(a)$ and for all Froude numbers $(b)$ shown in figure 4. In $(a)$, the black thin curve corresponds to the vertical spectrum $E_{\left[K, \kappa_{h} \leqslant k_{b}\right]}\left(k_{z}\right)$ computed with modes for which $\kappa_{h} \leqslant k_{b}$ and the dashed curve to the spectrum $E_{\left[K, \kappa_{h}>k_{b}\right]}\left(k_{z}\right)$ computed with modes for which $\kappa_{h}>k_{b}$. The dotted lines indicate the Ozmidov wavenumber $k_{z}=k_{o}$. The dotted dashed lines show the $k_{z}^{-3}$ power law. In $(b)$, the solid curves correspond to the spectra $E_{\left[K, \kappa_{h} \leqslant k_{b}\right]}\left(k_{z}\right)$ and the dashed curves to the spectra $E_{\left[K, \kappa_{h}>k_{b}\right]}\left(k_{z}\right)$.

has to be pointed out that the constant $C=0.56$ associated with small-scale turbulence is smaller than the classical Kolmogorov constant for the one-dimensional kinetic energy spectrum of homogeneous isotropic turbulence $C_{K} \simeq 1$ (Monin \& Yaglom 1975; Sreenivasan 1995; Gotoh, Fukayama \& Nakano 2002). However, the precise value of the constant $C$ is not meaningful here and depends on the horizontal size of the numerical box because the turbulence does not invade the whole computational domain.

Figure 6(a) presents a decomposition of the vertical compensated spectra $E_{K}\left(k_{z}\right) \varepsilon_{K}^{-2 / 3} k_{z}^{5 / 3}$ for $F_{h}=0.09$. The continuous thin curve corresponds to the conditional vertical spectrum $E_{\left[K, \kappa_{h} \leqslant k_{b}\right]}\left(k_{z}\right)$ computed with modes for which $\kappa_{h} \leqslant k_{b}$. As shown in figure 1, these modes correspond to the dipole deformed by the zigzag instability. This conditional vertical spectrum is very steep and clearly dominates the total spectrum at the largest vertical scales where the $N^{2} k_{z}^{-3}$ power law is observed. The dashed curve corresponds to the conditional vertical spectrum $E_{\left[K, \kappa_{h}>k_{b}\right]}\left(k_{z}\right)$ computed with modes for which $\kappa_{h}>k_{b}$. We have shown in figure 1 that these scales are generated mostly through the $\mathrm{KH}$ instability. We see that this conditional vertical spectrum is nearly flat from $k_{z} \simeq 3 k_{b}$ to the dissipative range, i.e. the range corresponding to a $k_{z}^{-5 / 3}$ power law. This spectrum does not show any tendency to steepen at the largest vertical scales. This indicates that the turbulent structures generated through the shear instability are relatively isotropic with a $k^{-5 / 3}$ inertial range. This is consistent with the relative isotropy of the $\mathrm{KH}$ billows seen in figure 1, where the peak corresponding to these structures is close to (and even below) the diagonal isotropic line $\kappa_{h}=k_{z}$ (see also the visualizations of the KH billows in figure 7 of Deloncle et al. 2008). This feature is hidden in the total vertical kinetic energy spectra at the large vertical scales between the buoyancy and the Ozmidov length scales owing to the dominance of the $N^{2} k_{z}^{-3}$ spectra associated with the large horizontal scales. The conditional spectra for the four Froude numbers are plotted in figure $6(b)$ as a function of $k_{z} / k_{o}$. This shows that the beginning of the $k_{z}^{-5 / 3}$ 


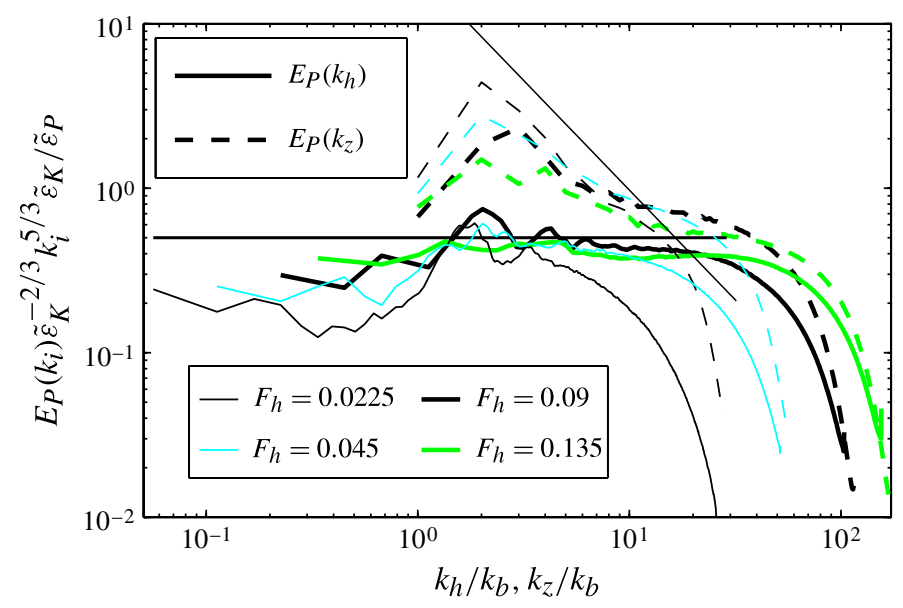

FIGURE 7. (Colour online) Similar to figure 4 except that it is for the spectra of potential energy. The horizontal line shows the $0.5 \tilde{\varepsilon}_{K}^{2 / 3} k^{-5 / 3} \varepsilon_{P} / \varepsilon_{K}$ law.

inertial range associated with small horizontal scales $\kappa_{h}>k_{b}$ scales with the Ozmidov wavenumber.

Figure 7 presents the horizontal (continuous lines) and vertical (dashed lines) compensated spectra $E_{P}\left(k_{i}\right) \tilde{\varepsilon}_{K}{ }^{2 / 3} k_{i}^{5 / 3} \varepsilon_{K} / \varepsilon_{P}$ of potential energy for the four Froude numbers. An average has been performed over the same time interval as in figure 4 . The potential spectra are very similar to the kinetic spectra (figure 4) but with less energy at large horizontal scales since the initial dipole has no potential energy. More pronounced bumps around the buoyancy length scale can be also seen. Like the horizontal compensated kinetic energy spectra $E_{K}\left(k_{h}\right) \tilde{\varepsilon}_{K}{ }^{2 / 3} k_{h}{ }^{5 / 3}$ (figure 4), the horizontal compensated potential energy spectra $E_{P}\left(k_{h}\right) \tilde{\varepsilon}_{K}{ }^{2 / 3} k_{h}{ }^{5 / 3} \varepsilon_{K} / \varepsilon_{P}$ present a flat range corresponding to a $k_{h}^{-5 / 3}$ power law from the buoyancy wavenumber to the dissipative wavenumber and nearly collapse at a value approximately equal to 0.5 . This means that the relation $E_{P}\left(k_{h}\right) / \varepsilon_{P}=E_{K}\left(k_{h}\right) / \varepsilon_{K}$ approximately holds as observed in forced strongly stratified turbulence (Lindborg 2006; Brethouwer et al. 2007). However, the compensated spectra are slightly lower than predicted and slightly decrease over the inertial range before the dissipative range.

The total dissipation is plotted versus time in figure $8(a)$. For all Froude numbers, we see an increase at $t \simeq 3.7$ corresponding to the development of the KH instability. The dissipation before this time is mostly due to the vertical shear resulting from the development of the zigzag instability. It is thus proportional to the inverse of the buoyancy Reynolds number $\left(\varepsilon \sim v\left(U / L_{b}\right)^{2} \sim U^{3} /\left(L_{h} \mathscr{R}\right)\right)$. Interestingly, when the horizontal Froude number is increased, the dissipation peak tends to last a longer time, i.e. to be broader. This might be related to the turn-over time scale of the KH billows which is likely to scale like $1 / N$ since their typical velocity is $U$ and their size $U / N$. Checking this hypothesis would require further investigations.

Figure $8(b)$ presents the temporal evolution of the instantaneous mixing efficiency $\Gamma(t) \equiv \varepsilon_{P}(t) / \varepsilon_{K}(t)$. We see that the mixing efficiency is around 0.4 and weakly varies with the stratification and with the time period during which the dissipation is strong. We have also looked at the isotropy of the dissipation by considering the ratio $\varepsilon_{z}(t) / \varepsilon(t)$ where $\varepsilon_{z}$ is the dissipation due to vertical gradients. The maximum 

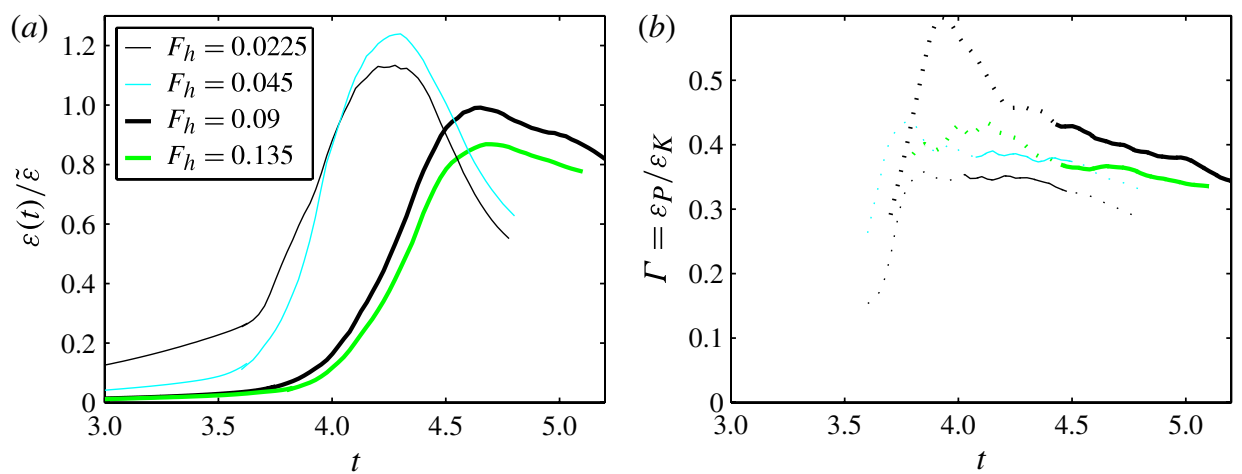

FIgURE 8. (Colour online) Temporal evolution of $(a)$ the total dissipation rate $\varepsilon(t)$ for $F_{h}=0.0025, F_{h}=0.0045, F_{h}=0.09, F_{h}=0.135$ scaled by $\tilde{\varepsilon}$ the maximum dissipation rate for $F_{h}=0.09$ and $(b)$ the mixing efficiency $\Gamma=\varepsilon_{P}(t) / \varepsilon_{K}(t)$ (continuous lines are used when the dissipation rate is high $\varepsilon(t)>0.8 \max \varepsilon(t))$.

value of $\varepsilon_{z} / \varepsilon$ increases with $\mathscr{R}$ and tends to $1 / 3$, which corresponds to an isotropic dissipation (not shown) even though a weak isotropic hyperviscosity is used.

\section{Effects of the Reynolds number and of the resolution for $F_{h}=0.09$}

We now focus on the effects of the variation of the Reynolds number and of the resolution. Two additional simulations have been carried out for $F_{h}=0.09$ and for the intermediate resolution $N_{h}=N_{x}=N_{y}=768$ and $N_{z}=192$, but for different values of the Reynolds number $R e=14000$ and $R e=7000$. Figure 9(a) displays the horizontal compensated spectra $E_{K}\left(k_{h}\right) \tilde{\varepsilon}_{K}^{2 / 3} k_{h}^{5 / 3}$ for these two simulations with a lower Reynolds number and for the other simulations with $R e=28000$ and intermediate $\left(N_{h}=768\right)$, large $\left(N_{h}=1024\right)$ and very large $\left(N_{h}=1280\right)$ resolutions (see table 1$)$. We see that the width of the inertial range strongly decreases when the Reynolds number is decreased. However, the bump corresponding to the shear instability is almost unaffected meaning that, when the Reynolds number and the buoyancy Reynolds number are large enough, the horizontal wavelength of the shear instability continues to scale with the buoyancy length scale. In addition, as already stated, there are almost no differences between the two spectra for $N_{h}=1024$ and $N_{h}=1280$ except at the smallest scales of the dissipative range, validating the use of a weak isotropic hyperviscosity.

The total dissipation (continuous lines) and the hyperdissipation (dashed lines) are plotted versus time for the same runs in figure $9(b)$. We see that despite the important variation of resolution, the total dissipation curves for $R e=28000$ (black thick and thin lines and light thick line) are quite close, especially for the two largest resolution simulations (thick lines). In contrast, the hyperdissipation strongly decreases when the resolution is increased indicating that the Kolmogorov scale becomes nearly resolved. For the lowest Reynolds number $R e=7000$, there is no need for hyperviscosity and the simulation is a real DNS. The total dissipation curves for the different Reynolds numbers slightly differ. For lower $R e$, the dissipation is more important during the nonlinear evolution of the zigzag instability before the development of the secondary instabilities that occurs after $t=3.7$. The increase corresponding to the development of the secondary instabilities is slightly slower leading to a slightly lower maximum of total dissipation of the order of $0.9 \tilde{\varepsilon}$ for $R e=7000$ and $0.94 \tilde{\varepsilon}$ for $R e=14000$. 

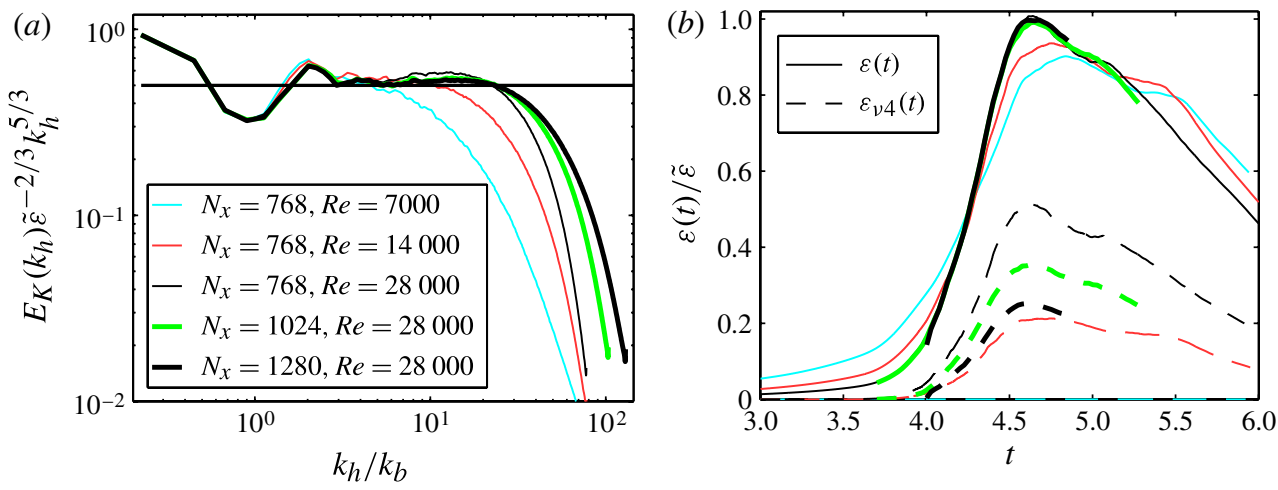

FIGURE 9. (Colour online) (a) Horizontal compensated one-dimensional spectra $E_{K}\left(k_{h}\right)$ $\tilde{\varepsilon}_{K}^{2 / 3} k_{h}{ }^{5 / 3}$ for five runs for different values of the Reynolds number and of resolution but for the same Froude number $F_{h}=0.09$. Each curve is the average over a time interval $\Delta t=0.3$ near the maximum of the dissipation. The horizontal line shows the $0.5 \tilde{\varepsilon}_{K}^{2 / 3} k_{h}^{-5 / 3}$ law. (b) Temporal evolution of the total dissipation rate $\varepsilon(t)$ (continuous lines) and of the hyperdissipation rate $\varepsilon_{v_{4}}(t)$ (dashed lines) scaled by $\tilde{\varepsilon}$, the maximum dissipation rate for $R e=28000$, and the maximum resolution $N_{h}=1280$.

However, the global evolution of the total dissipation rate is only weakly affected by the variation of the Reynolds number. This indicates that the first mechanisms of the transition to turbulence, namely the nonlinear evolution of the zigzag instability and the secondary instabilities, are only weakly influenced by dissipation for these values of the buoyancy Reynolds number and the Reynolds number.

\section{Decomposition of the horizontal fluxes for $F_{h}=0.09$}

The evolution equations of the kinetic and potential energies $\hat{E}_{K}(\boldsymbol{k})=|\hat{\boldsymbol{u}}|^{2} / 2$ and $\hat{E}_{P}(\boldsymbol{k})=\left|\hat{\rho}^{\prime}\right|^{2} /\left(2 F_{h}^{2}\right)$ of a wavenumber $\boldsymbol{k}$ can be expressed as

$$
\begin{aligned}
& \frac{\mathrm{d} \hat{E}_{K}(\boldsymbol{k})}{\mathrm{d} t}=\hat{T}_{K}-\hat{b}-\hat{D}_{K}, \\
& \frac{\mathrm{d} \hat{E}_{P}(\boldsymbol{k})}{\mathrm{d} t}=\hat{T}_{P}+\hat{b}-\hat{D}_{P},
\end{aligned}
$$

where $\hat{T}_{K}=-\operatorname{Re}\left\{\hat{\boldsymbol{u}}^{*}(\boldsymbol{k}) \cdot P_{\perp}(\widehat{\boldsymbol{u} \cdot \nabla \boldsymbol{u}})(\boldsymbol{k})\right\}$ and $\hat{T}_{P}=-F_{h}^{-2} \operatorname{Re}\left\{\hat{\rho}^{\prime *}(\boldsymbol{k})\left(\widehat{\boldsymbol{u} \cdot \nabla \rho^{\prime}}\right)(\boldsymbol{k})\right\}$ are the kinetic and potential nonlinear transfers, $\hat{D}_{K}(\boldsymbol{k})=\left(|\boldsymbol{k}|^{2} / R e+|\boldsymbol{k}|^{8} / \operatorname{Re}_{4}\right)|\hat{\boldsymbol{u}}|^{2}$ and $\hat{D}_{P}(\boldsymbol{k})=\left(|\boldsymbol{k}|^{2} /(\operatorname{ReSc})+|\boldsymbol{k}|^{8} / R e_{4}\right)\left|\hat{\rho}^{\prime}\right|^{2} / F_{h}{ }^{2}$ are the kinetic and potential mean energy dissipation and $\hat{b}(\boldsymbol{k})=F_{h}^{-2} \operatorname{Re}\left\{{\hat{\rho^{\prime}}}^{*}(\boldsymbol{k}) \hat{w}(\boldsymbol{k})\right\}$ is the local (in spectral space) conversion of kinetic energy into potential energy. When (6.1) and (6.2) are summed over the wavenumbers inside a vertical cylinder $\Omega_{\kappa_{h}}$ of radius $\kappa_{h}$ in spectral space, we obtain

$$
\begin{aligned}
& \frac{\mathrm{d} \mathscr{E}_{K}\left(\kappa_{h}\right)}{\mathrm{d} t}=-\Pi_{K}\left(\kappa_{h}\right)-\mathscr{C}\left(\kappa_{h}\right)-\varepsilon_{K}\left(\kappa_{h}\right), \\
& \frac{\mathrm{d} \mathscr{E}_{P}\left(\kappa_{h}\right)}{\mathrm{d} t}=-\Pi_{P}\left(\kappa_{h}\right)+\mathscr{C}\left(\kappa_{h}\right)-\varepsilon_{P}\left(\kappa_{h}\right),
\end{aligned}
$$



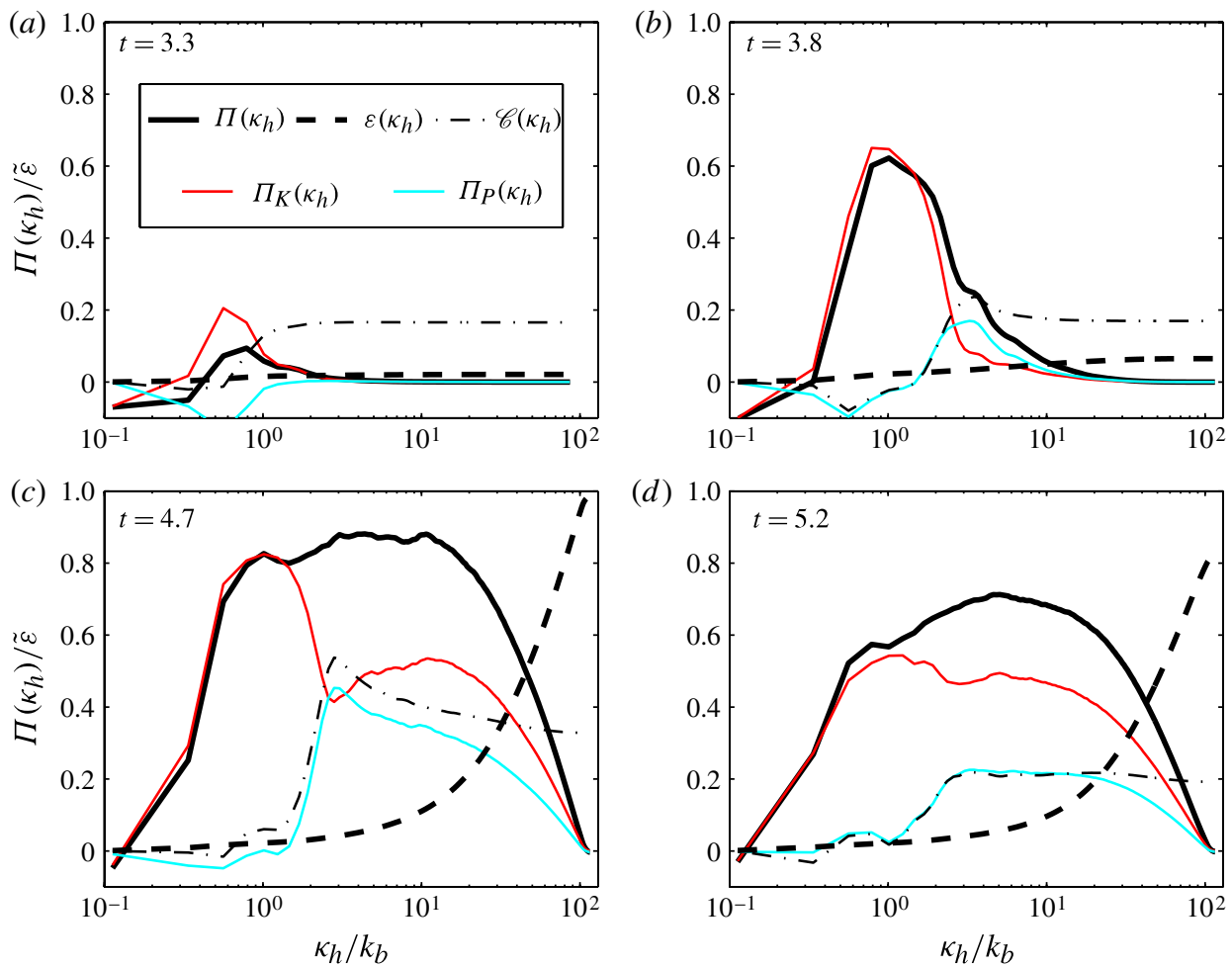

FIGURE 10. (Colour online) Fluxes going out from a vertical cylinder $\Omega_{\kappa_{h}}$ of radius $\kappa_{h}$ in spectral space and dissipations inside this cylinder. The continuous darker grey thin, light grey thin and black thick curves are respectively the kinetic $\Pi_{K}\left(\kappa_{h}\right)$, potential $\Pi_{P}\left(\kappa_{h}\right)$ and total horizontal fluxes through the surface of $\Omega_{\kappa_{h}}$. The dashed thick curve is the total cumulative dissipation inside the volume $\Omega_{\kappa_{h}}$. The dotted-dashed black curve is $\mathscr{C}\left(\kappa_{h}\right)$, the cumulative conversion from kinetic into potential energies, i.e. the sum inside the volume $\Omega_{\kappa_{h}}$ of the local conversion $\hat{b}(\boldsymbol{k})$. The lowest wavenumber corresponds to the shear modes.

where $\mathscr{E}_{K}\left(\kappa_{h}\right)=\sum_{\left|k_{h}\right| \leqslant \kappa_{h}, k_{z}} \hat{E}_{K}(\boldsymbol{k}), \Pi_{K}\left(\kappa_{h}\right)$ is the kinetic flux going out of $\Omega_{\kappa_{h}}, \mathscr{C}\left(\kappa_{h}\right)$ the cumulative conversion rate of kinetic energy into potential energy inside $\Omega_{\kappa_{h}}$ and $\varepsilon_{K}\left(\kappa_{h}\right)$ the cumulative kinetic dissipation rate inside $\Omega_{\kappa_{h}}$. The quantities with the subscript $P$ are defined similarly but for the potential energy. In order for the fluxes of the shear modes not to be located at $-\infty$ in logarithmic plots, the horizontal wavenumber $\kappa_{h}$ is discretized as $\kappa_{h}=\delta \kappa_{h} / 2+\delta \kappa_{h} l$, where $\delta \kappa_{h}=2 \pi / \mathscr{L}_{h}$ and $l$ is the discretization integer. It is convenient to consider the integrated (6.3)-(6.4) because the nonlinear terms are conservative and dissipation is non-negligible only at small scales. This implies that the spectral fluxes (and dissipation rates) are fundamental quantities like in isotropic turbulence (Kolmogorov 1941). Similarly, it is convenient to consider the cumulative conversion rate $\mathscr{C}\left(\kappa_{h}\right)$ from kinetic to potential energies because the variations of this quantity as a function of wavenumber can be directly compared with the maximum dissipation rate.

The energy fluxes and cumulative conversion and dissipation rates are plotted versus $\kappa_{h}$ for four particular times in figure 10 for $F_{h}=0.09$ and $R e=28000$. All the curves have been scaled by $\tilde{\varepsilon}$, the maximum of the total instantaneous dissipation. The plot for $t=3.3$ (figure $10 a$ ) corresponds to a time at which the zigzag instability 
evolves nonlinearly but the shear instability has not yet developed. At this time all the quantities are small compared to $\tilde{\varepsilon}$. The dissipation (dashed line) is negligible. There is only a weak kinetic energy flux (darker grey thin continuous line) of order $0.2 \tilde{\varepsilon}$ toward horizontal wavenumbers slightly larger than the leading horizontal wavenumber of the two-dimensional base flow $k_{0}$ (which is of order $0.4 k_{b}$ for the particular stratification $F_{h}=0.09$ ). By looking at the flux for other $F_{h}$, we have observed that the horizontal wavenumbers at which kinetic energy is transferred at this time do not scale as the buoyancy wavenumber but as $k_{0}$. The weakness of the flux along the horizontal when only the zigzag instability is active is consistent with figure 1, which shows that the zigzag instability produces a strong flux along the vertical toward large vertical wavenumber of order $k_{b}$ but only a weak flux along the horizontal. At wavenumbers slightly larger than $k_{0}$, the cumulative conversion $\mathscr{C}\left(\kappa_{h}\right)$ increases from nearly zero to approximately $0.17 \tilde{\varepsilon}$. Since $\mathscr{C}\left(\kappa_{h}\right)=\sum_{\left|k_{h}\right| \leqslant \kappa_{h}, k_{z}} \hat{b}(\boldsymbol{k})$, an increase (respectively decrease) of $\mathscr{C}\left(\kappa_{h}\right)$ indicates positive (respectively negative) local conversion of kinetic into potential energies. The variation $\Delta \mathscr{C}\left(\Delta \kappa_{h}\right)$ of the cumulative conversion over a particular range of wavenumbers $\Delta \kappa_{h}$ represents the energy converted over this range. In this case, in the range of wavenumber $\Delta \kappa_{h}=\left[0.6 k_{b}, k_{b}\right]$ around $2 k_{0}$, we have $\Delta \mathscr{C}\left(\Delta \kappa_{h}\right) \simeq 0.17 \tilde{\varepsilon}$. This conversion at wavenumbers $\kappa_{h} \simeq 2 k_{0}$ is due to the bending of the vortices. Remarkably, there is also a backward potential energy flux (light continuous line) toward the smallest horizontal wavenumbers of the numerical box. However, the potential energy flux toward the horizontally invariant 'shear modes' (located at the first point $\kappa_{h} \simeq 0.1 k_{b}$ as previously explained) is zero. In contrast, the kinetic energy flux is negative for the smallest wavenumber $\kappa_{h} \simeq 0.1 k_{b}$, indicating a flux to shear modes of order $0.06 \tilde{\varepsilon}$.

The time $t=3.8$ (figure $10 b$ ) corresponds to the development of the KH billows before the transition to turbulence. As for $t=3.3$, the dissipation (dashed lines) is negligible and there is a weak kinetic energy flux toward shear modes. The potential flux is still negative at large horizontal scales $0.1 \leqslant \kappa_{h} / k_{b} \lesssim 1$ and is now balanced by the cumulative energy conversion $\mathscr{C}\left(\kappa_{h}\right)$. This shows that there is almost no more accumulation of potential energy at large scales since potential energy transferred at these scales is now converted to kinetic energy. The kinetic energy flux becomes positive at $\kappa_{h} \simeq 0.3 k_{b}$ (second point), reaches a maximum around $\kappa_{h}=0.8 k_{b}$, and drops down to nearly zero around $\kappa_{h} \simeq 3 k_{b}$. This means that the kinetic energy is transferred from the large scales $\kappa_{h} \simeq k_{0}$ toward horizontal scales around $\kappa_{h}=2 k_{b}$. This flux appears as a peak and not as a plateau because the ratio $2 k_{b} / k_{0}$ is not large but only moderate for $F_{h}=0.09$. However, the other runs for lower $F_{h}$ (not plotted) show that for the kinetic energy flux this non-local transfer appears as a plateau with a width $2 k_{b} / k_{0}$ proportional to $F_{h}^{-1}$. The cumulative conversion of kinetic into potential energies (black dashed-dotted curve) becomes positive at horizontal wavenumber $\kappa_{h} \simeq 1.5 k_{b}$, reaches its maximum at $\kappa_{h} \simeq 3 k_{b}$ to $4 k_{b}$, and then slightly decreases and remains constant around $0.2 \tilde{\varepsilon}$ for smaller scales. Again, the increase of $\mathscr{C}\left(\kappa_{h}\right)$ at $k_{b}$ is due to the development of the $\mathrm{KH}$ billows, which convert kinetic energy into potential energy at the buoyancy length scale. The slight decrease of $\mathscr{C}\left(\kappa_{h}\right)$ at smaller scales corresponds to a weak local conversion of potential energy back into kinetic energy. The constant cumulative conversion $\mathscr{C}\left(\kappa_{h}\right)$ at $\kappa_{h}>10 k_{b}$ indicates that there is no local conversion in this wavenumber range.

Figure $10(c)$ corresponds to the time $t=4.7$ when the dissipation is maximum. The total dissipation $\varepsilon\left(\kappa_{h}\right)$ (thick dashed line) reaches the value $\tilde{\varepsilon}$ for the largest $\kappa_{h}$. The kinetic energy flux at large scales is similar to the one for $t=3.8$ with an even 
stronger flux from large scales toward $\kappa_{h} \simeq 2 k_{b}$. The peak at large scales corresponding to the development of the $\mathrm{KH}$ billows has reached a value close to unity just before at $t=4.5$. The increase of cumulative conversion of kinetic into potential energies at wavenumbers $\kappa_{h} \simeq 2 k_{b}$ is now much larger $(\Delta \mathscr{C} \simeq 0.5 \tilde{\varepsilon})$ indicating that the KH billows are efficient in displacing isopycnals. At wavenumbers around $\kappa_{h} \simeq 3 k_{b}$, kinetic and potential fluxes are nearly equal. In contrast to figure $10(b)$, the total flux, equal to $0.8 \tilde{\varepsilon}$ between $k_{0}$ and $2 k_{b}$, does not drop to zero but reaches another plateau close to $\Pi\left(\kappa_{h}\right)=0.9 \tilde{\varepsilon}$ down to the dissipation range. This second plateau at small scales is due to different processes than the peak at large scales, namely the destabilization of the $\mathrm{KH}$ billows and the gravitational instability. At small scales, there is a local conversion of potential energy back into kinetic energy (the dotted-dashed curve $\mathscr{C}\left(\kappa_{h}\right)$ goes down), which is driven by these instabilities and the associated transition to turbulence. This conversion, accounting for $\Delta \mathscr{C} \simeq-0.2 \tilde{\varepsilon}$, leads to an increase of the kinetic energy flux and a decrease of potential energy flux (light continuous curve) with a nearly constant total energy flux. In addition, the upscale energy fluxes at large scales have significantly decreased.

At later time $t=5.2$ (figure $10 d$ ), the dissipation is still close to $0.8 \tilde{\varepsilon}$ but the plateau of kinetic energy flux has decreased to $0.5 \tilde{\varepsilon}$. The new feature is that the total flux at small scales is now dominated by the kinetic energy flux, the potential energy flux being nearly 2 times smaller. This may be the sign of a restratification with weaker overturning events. Moreover, both potential flux and cumulative conversion are remarkably flat from $\kappa_{h} \simeq 3 k_{b}$ to the dissipative range, meaning that there is a constant potential flux with no local energy conversion. This may indicate that the density at small scales is passively advected during the late decay.

Since the initial flow has no potential energy and since the energy conversion at large scales is weak during the lifetime of the dipole, no potential energy is available at large scales and the cascade of potential energy toward small scales should exist at the expense of the kinetic energy. This is demonstrated by the fact that potential energy flux $\Pi_{P}\left(\kappa_{h}\right)$ is almost always equal to the cumulative conversion of kinetic energy into potential energy $\mathscr{C}\left(\kappa_{h}\right)$. Remarkably, $\mathscr{C}\left(\kappa_{h}\right)$ always becomes positive when $\kappa_{h} \gtrsim k_{b}$, i.e. for the scales created by the development of secondary instabilities.

\section{Summary and conclusions}

We have presented a spectral analysis of the transition to turbulence from a columnar dipole in a stratified fluid. A series of instabilities and nonlinear processes occurs in a particular time sequence leading to a breakdown into small-scale turbulence.

We have shown that the transition to turbulence occurring during the nonlinear evolution of the zigzag instability has a two-step dynamics. First, a shear instability feeds quasi-isotropic and fast Kelvin-Helmholtz billows with a vertical Froude number of order unity and a typical scale of the order of the buoyancy scale, i.e. larger than the Ozmidov length scale. Second, the destabilization of these structures and the gravitational instability generate turbulence from the buoyancy scale to the dissipative range. This turbulent regime is weakly stratified because the associated larger structures are roughly isotropic (with horizontal and vertical characteristic length scales of the same order) and are linked to vertical and horizontal Froude numbers of order unity. Moreover, significant vertical motions due to overturnings exist in this regime. 
The spectra have been shown to be strongly anisotropic. The horizontal spectra exhibit a $k_{h}^{-5 / 3}$ inertial range. Nevertheless, there is a deficit of energy in the range between the large scales associated with the dipole and the buoyancy length scale. Remarkably, at smaller scales and down to the dissipative scales, the kinetic and potential energy horizontal spectra approximately collapse on the $0.5 \varepsilon_{K}{ }^{2 / 3} k_{h}^{-5 / 3}$ and $0.5 \varepsilon_{K}^{2 / 3} k_{h}^{-5 / 3} \varepsilon_{P} / \varepsilon_{K}$ spectra, respectively. Thus the relation $E_{P}\left(k_{h}\right) / \varepsilon_{P}=E_{K}\left(k_{h}\right) / \varepsilon_{K}$ approximately holds as measured in numerical simulations of forced stratified turbulence. The vertical kinetic spectrum follows a $C_{N} N^{2} k_{z}^{-3}$ law at large vertical scales, with $C_{N} \simeq 0.08$, which is due to the nonlinear evolution of the zigzag instability. For the largest values of the buoyancy Reynolds number $\mathscr{R}$, the vertical spectrum presents a transition at the Ozmidov length scale $l_{o}$ toward a $C \varepsilon_{K}{ }^{2 / 3} k^{-5 / 3}$ spectrum, with $C \simeq 0.56$.

Thus, the anisotropic spectra share many characteristics with those obtained from numerical simulations of forced stratified turbulence and from measurements in the atmosphere and in the ocean. This is remarkable because the initial flow is very simple and not turbulent. Moreover, the fundamental difference between a transition toward turbulence and developed turbulence has to be stressed. With only two vortices interacting, the dynamics at large horizontal scales is dominated by the zigzag instability and there is no strongly stratified cascade along the horizontal. This contrasts with numerical simulations of forced stratified turbulence which exhibit a forward strongly stratified cascade but for which the overturning motions at the buoyancy length scale and beyond are not resolved or only weakly resolved due to the use of strongly anisotropic numerical meshes (see e.g. Koshyk \& Hamilton 2001; Lindborg 2006; Waite 2011).

Since the transition in the vertical spectra happens at the Ozmidov length scale, it is tempting to conclude that the overturning motions at the buoyancy scale are strongly anisotropic. However, this is not the case. Indeed, we have shown that the very steep vertical spectrum is mainly due to the large horizontal scales of the dipole that is strongly deformed along the vertical by the zigzag instability. In contrast, the vertical spectrum computed with spectral modes with horizontal wavenumbers larger than the buoyancy wavenumber $k_{b}$ does not present any $k_{z}^{-3}$ power law but exhibits a $k_{z}^{-5 / 3}$ power law from a vertical wavenumber scaling like the Ozmidov wavenumber $k_{o}$ down to the dissipative range.

In this paper, we have stressed the qualitative difference between the buoyancy length scale $L_{b}$ and the Ozmidov length scale $l_{o}$. However, quantitatively, the ratio $L_{b} / l_{o}$ scales like $F_{h}^{-1 / 2}$ and is therefore not very large for $F_{h}=O(0.1)$. In the present case, the Ozmidov wavenumber can be computed as

$$
k_{o}=\left(\frac{C_{N}}{C}\right)^{3 / 4}\left(\frac{N^{3}}{\varepsilon_{K}}\right)^{1 / 2}=F_{h}^{-3 / 2}\left(\frac{C_{N}}{C}\right)^{3 / 4}\left(\frac{U^{3}}{\varepsilon_{K} R}\right)^{1 / 2} \frac{1}{R},
$$

and the buoyancy wavenumber is $k_{b}=2 \pi /\left(10 F_{h}\right)$. The ratio is therefore

$$
\frac{k_{o}}{k_{b}}=F_{h}^{-1 / 2}\left(\frac{C_{N}}{C}\right)^{3 / 4}\left(\frac{U^{3}}{\varepsilon_{K} R}\right)^{1 / 2} \frac{10}{2 \pi} \simeq 1.4 F_{h}^{-1 / 2},
$$

and varies only from 9.3 to 3.8 when $F_{h}$ increases from 0.0225 to 0.135 .

It has to be pointed out that recent results highlight the importance of the buoyancy length scale on forced stratified turbulence (Waite 2011). Finally, we can conjecture that such non-local transfers due to secondary instabilities act as a leak from the 


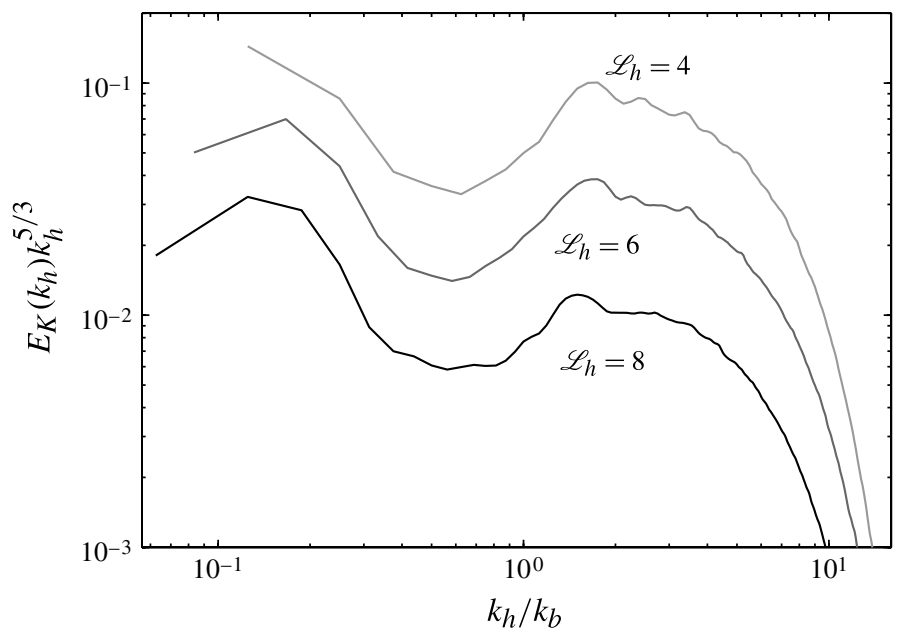

FIGURE 11. Horizontal compensated spectra $E_{K}\left(k_{h}\right) k_{h}{ }^{5 / 3}$ for three runs for different values of the horizontal size of the numerical domain $\mathscr{L}_{h}=4,6$ and 8 but for the same grid spacing $4 / 256=6 / 384=8 / 512$, Reynolds number $R e=10000$ and Froude number $F_{h}=0.05$.

strongly stratified turbulent cascade toward a weakly stratified turbulence beyond the buoyancy scale. However, the horizontal scales larger than the buoyancy length scale dominate the vertical spectra down to the Ozmidov length scale.

\section{Appendix. Effect of the horizontal size of the numerical domain}

In order to address the effect of the horizontal size of the numerical domain $\mathscr{L}_{h}$, we have carried out three simulations for $\mathscr{L}_{h}=4,6$ and 8 keeping the same grid spacing (the number of nodes in one horizontal direction $N_{h}$ is equal to 256, 384 and 512, respectively) and the same physical parameters: Reynolds number $R e=10000$ and Froude number $F_{h}=0.05$. Note that the resolution and the Reynolds number are much smaller than for the simulations presented in the body of the paper. As already mentioned, we have verified that the transition to turbulence involves the same mechanisms for all the simulations.

Figure 11 shows the horizontal compensated spectra $E_{K}\left(k_{h}\right) k_{h}^{5 / 3}$ at the maximum of dissipation. The magnitude of the spectra decreases with $\mathscr{L}_{h}$ since turbulence invades a smaller part of the numerical domain for larger numerical boxes. At the smallest wavenumbers, their shape significantly varies with $\mathscr{L}_{h}$. Apart from these differences, the spectra at larger wavenumbers are very similar. This confirms that our results are only weakly sensitive to the size of the numerical domain and validates our choice of using a small numerical domain in order to resolve smaller scales and simulate larger Reynolds numbers.

\section{REFERENCES}

AUgIER, P. 2011 Turbulence in strongly stratified fluids: cascade processes. PhD thesis, LadHyX, Ecole Polytechnique. http://tel.archives-ouvertes.fr/tel-00697245.

Augier, P. \& Billant, P. 2011 Onset of secondary instabilities on the zigzag instability in stratified fluids. J. Fluid Mech. 662, 120-131.

BILlant, P. 2010 Zigzag instability of vortex pairs in stratified and rotating fluids. Part 1. General stability equations. J. Fluid Mech. 660, 354-395. 
Billant, P. \& Chomaz, J.-M. 2000a Experimental evidence for a new instability of a vertical columnar vortex pair in a strongly stratified fluid. J. Fluid Mech. 418, 167-188.

Billant, P. \& Chomaz, J.-M. $2000 b$ Theoretical analysis of the zigzag instability of a vertical columnar vortex pair in a strongly stratified fluid. J. Fluid Mech. 419, 29-63.

BILlant, P. \& Chomaz, J.-M. 2000c Three-dimensional stability of a vertical columnar vortex pair in a stratified fluid. J. Fluid Mech. 419, 65-91.

Billant, P. \& Chomaz, J.-M. 2001 Self-similarity of strongly stratified inviscid flows. Phys. Fluids 13, 1645-1651.

Billant, P., Deloncle, A., Chomaz, J.-M. \& Otheguy, P. 2010 Zigzag instability of vortex pairs in stratified and rotating fluids. Part 2. Analytical and numerical analyses. J. Fluid Mech. 660, 396-429.

Brethouwer, G., Billant, P., Lindborg, E. \& Chomaz, J.-M. 2007 Scaling analysis and simulation of strongly stratified turbulent flows. J. Fluid Mech. 585, 343-368.

DE BRUYN Kops, S. M. \& RILEY, J. J. 1998 Direct numerical simulation of laboratory experiments in isotropic turbulence. Phys. Fluids 10, 2125-2127.

CAmbon, C. 2001 Turbulence and vortex structures in rotating and stratified flows. Eur. J. Mech. (B/Fluids 20, 489-510.

CRAYA, A. D. 1958 Contribution à l'analyse de la turbulence associée à des vitesses moyennes. Ministére de l'air, France PST 345.

Deloncle, A., Billant, P. \& Chomaz, J.-M. 2008 Nonlinear evolution of the zigzag instability in stratified fluids: a shortcut on the route to dissipation. J. Fluid Mech. 599, 229-238.

DEWAN, E. 1997 Saturated-cascade similitude theory of gravity wave spectra. J. Geophys. Res. 102 (D25), 29799-29817.

Garrett, C. \& Munk, W. 1979 Internal waves in the ocean. Annu. Rev. Fluid Mech. 11, 339-369.

Godeferd, F. S. \& Staquet, C. 2003 Statistical modelling and direct numerical simulations of decaying stably stratified turbulence. Part 2. Large-scale and small-scale anisotropy. J. Fluid Mech. 486, 115-159.

Gotoh, T., FukAYAma, D. \& NAKAno, T. 2002 Velocity field statistics in homogeneous steady turbulence obtained using a high-resolution direct numerical simulation. Phys. Fluids 14 (3), $1065-1081$.

GregG, M. C. 1987 Diapycnal mixing in the thermocline - a review. J. Geophys. Res. 92 (C5), 5249-5286.

Hebert, D. A. \& DE BRUYn Kops, S. M. 2006 Predicting turbulence in flows with strong stable stratification. Phys. Fluids 18, 066602.

Herring, J. R. 1974 Approach of axisymmetric turbulence to isotropy. Phys. Fluids 17, 859-872.

Holloway, G. 1983 A conjecture relating oceanic internal waves and small-scale processes. Atmos.-Ocean 21 (1), 107-122.

Kolmogorov, A. N. 1941 The local structure of turbulence in incomressible viscous fluids for very large Reynolds numbers. C. R. Acad. Sci. (USSR) 30, 301-305.

KoshyK, J. N. \& HAmilton, K. 2001 The horizontal kinetic energy spectrum and spectral budget simulated by a high-resolution troposphere-stratosphere-mesosphere GCM. J. Atmos. Sci. 58 (4), 329-348.

Laval, J. P., McWilliams, J. C. \& Dubrulle, B. 2003 Forced stratified turbulence: successive transitions with Reynolds number. Phys. Rev. E 68 (3, Part 2), 036308.

LINDBORG, E. 2002 Strongly stratified turbulence: a special type of motion. In Advances in Turbulence IX, Proceedings of the Ninth European Turbulence Conference, Southampton.

Lindborg, E. 2006 The energy cascade in a strongly stratified fluid. J. Fluid Mech. 550, 207-242.

Lindborg, E. \& BRethouwer, G. 2007 Stratified turbulence forced in rotational and divergent modes. J. Fluid Mech. 586, 83-108.

LUMLEY, J. L. 1964 The spectrum of nearly inertial turbulence in a stably stratified fluid. J. Atmos. Sci. 21 (1), 99-102.

Lundbladh, A., Berlin, S., Skote, M., Hildings, C., Choi, J., Kim, J. \& Henningson, D. S. 1999 An efficient spectral method for simulation of incompressible flow over a flat plate. Trita-mek. Tech. Rep. 11, 265-335. 
Monin, A. S. \& Yaglom, A. M. 1975 Statistical Fluid Mechanics, vol. 2. MIT.

NAStRom, G. D. \& GAGE, K. S. 1985 A climatology of atmospheric wavenumber spectra of wind and temperature observed by commercial aircraft. J. Atmos. Sci. 42, 950-960.

Otheguy, P., Chomaz, J.-M. \& Billant, P. 2006 Elliptic and zigzag instabilities on co-rotating vertical vortices in a stratified fluid. J. Fluid Mech. 553, 253-272.

OzMIDOV, R. V. 1965 On the turbulent exchange in a stably stratified ocean. Izv. Akad. Sci. USSR, Atmos. Ocean. Phys. 1, 493-497.

RILEY, J. J. \& DE BRUYN KOPS, S. M. 2003 Dynamics of turbulence strongly influenced by buoyancy. Phys. Fluids 15 (7), 2047-2059.

Riley, J. J. \& LindBorG, E. 2008 Stratified turbulence: a possible interpretation of some geophysical turbulence measurements. J. Atmos. Sci. 65, 2416-2424.

SMith, L. M. \& WALEFFE, F. 2002 Generation of slow large scales in forced rotating stratified turbulence. J. Fluid Mech. 451, 145-168.

SReEnivasan, K. R. 1995 On the universality of the Kolmogorov constant. Phys. Fluids 7 (11), 2778-2784.

Staquet, C. \& Riley, J. J. 1989 On the velocity field associated with potential vorticity. Dyn. Atmos. Oceans 14, 93-123.

Waite, M. L. 2011 Stratified turbulence at the buoyancy scale. Phys. Fluids 23 (6), 066602.

WAite, M. L. \& BARTello, P. 2004 Stratified turbulence dominated by vortical motion. J. Fluid Mech. 517, 281-308.

Waite, M. L. \& SMolarkiewicz, P. K. 2008 Instability and breakdown of a vertical vortex pair in a strongly stratified fluid. J. Fluid Mech. 606, 239-273. 